\title{
1 Informing shigellosis prevention and control through pathogen genomics
}

2

3 Authors:

4 Rebecca J. Bengtsson ${ }^{1}$, Adam J. Simpkin², Caisey V. Pulford ${ }^{1,7}$, Ross Low ${ }^{3}$, David A. Rasko ${ }^{5}$, Daniel J.

5 Rigden $^{2}$, Neil Hall ${ }^{3,4}$, Eileen M. Barry ${ }^{6}$, Sharon M. Tennant ${ }^{6}$, Kate S. Baker ${ }^{1}$

6

7 Affiliations:

$8 \quad{ }^{1}$ Clinical Infection, Microbiology and Immunity, Institute of Infection, Veterinary and Ecological

9 Sciences, The University of Liverpool, UK

$10 \quad{ }^{2}$ Biochemistry and Systems Biology, Institute of Systems, Molecular and Systems Biology, The

11 University of Liverpool, UK

$12 \quad{ }^{3}$ Earlham Institute, Norwich Research Park, Norwich, UK

$13{ }^{4}$ School of Biological Sciences, University of East Anglia, Norwich, UK

$14{ }^{5}$ Department of Microbiology and Immunology, Institute for Genome Sciences, University of Maryland

15 School of Medicine, Baltimore, Maryland, USA

$16{ }^{6}$ Center for Vaccine Development and Global Health, University of Maryland School of Medicine,

17 Baltimore, Maryland, USA

$18{ }^{7}$ Blood Safety, Hepatitis, Sexually Transmitted Infections and HIV Service' National Infection Service, 19 Public Health England, London, UK

20

\section{Corresponding Author:}

22 Kate S. Baker

23

Email: kbaker@liverpool.ac.uk 
Abstract

Shigella spp. are the leading bacterial cause of severe childhood diarrhoea in low- and middle- income countries (LMIC), are increasingly antimicrobial resistant and have no licensed vaccine. We performed genomic analyses of 1246 systematically collected shigellae from seven LMIC to inform control and identify factors that could limit the effectiveness of current approaches. We found that S. sonnei contributes $\geq 20$-fold more disease than other Shigella species relative to its genomic diversity and highlight existing diversity and adaptative capacity among $S$. flexneri that may generate vaccine escape variants in $<6$ months. Furthermore, we show convergent evolution of resistance against the current recommended antimicrobial among shigellae. This demonstrates the urgent need to integrate existing genomic diversity into vaccine and treatment plans for Shigella, and other pathogens.

\section{Introduction}

Shigellosis is a diarrhoeal disease responsible for approximately 212,000 annual deaths and accounting for $13.2 \%$ of all diarrhoeal deaths globally (1). The Global Enteric Multicenter Study (GEMS) was a large case-control study conducted between 2007 and 2011, investigating the aetiology and burden of moderate-to-severe diarrhoea (MSD) in children less than five years old in low- and middle-income countries (LMICs) (2). GEMS revealed shigellosis as the leading bacterial cause of diarrhoeal illness in children, who represent a major target group for vaccination (3). The aetiological agents are Shigella, a Gram-negative genus comprised of S. flexneri, S. sonnei, S. boydii and S. dysenteriae, with the former two serotypes causing the majority (90\%) of attributable shigellosis in children in LMICs (3). Currently, the disease is primarily managed through supportive care and antimicrobial therapy. However, there has been an increase in antimicrobial resistance (AMR) among Shigella (4). Particularly concerning is the rise of resistance against the fluoroquinolone antimicrobial ciprofloxacin, the current World Health Organisation (WHO) recommended treatment, such that fluoroquinolone-resistant (FQR) Shigella is one of a dozen pathogens for which WHO notes new antimicrobial therapies are urgently needed (5). The high disease burden and increasing AMR of Shigella call for improvements in treatment and management options for shigellosis, and significant momentum has built to rise to this challenge. its development is the considerable genomic and phenotypic diversity of the organisms (6). The distinct 
the short to medium term serotype-specific immunity following infection (7-10). Hence, considerable efforts are focused on generating $\mathrm{O}$-antigen specific vaccines. However, with the exception of the single serotype $S$. sonnei, each species encompasses multiple diverse serotypes: 14 serotypes/subserotypes for $S$. flexneri, 19 for $S$. boydii and 15 for $S$. dysenteriae (11). Thus, for serotype-targeted vaccine approaches, multivalent vaccines are proposed to provide broad protection against disease $(6,12)$. Furthermore, while O-antigen conjugates are a leading strategy, challenge studies have recently demonstrated poor efficacy $(13,14)$. An attractive alternative and/or complement to serotype-targeted vaccine formulations are specific subunit vaccines which target highly conserved proteins and may offer broad protection. There are several candidates in development that have demonstrated protection in animal models $(15,16)$, but the degree of antigenic variation for these targets among the global Shigella population remains unknown.

Whole-genome sequencing analysis (WGSA) provides sufficient discriminatory power to resolve phylogenetic relationships and characterise diversity of bacterial pathogens, essential to informing vaccine development and other aspects of disease control $(17,18)$. However, these critical analysis tools are yet to be applied to a pathogen collection appropriate for broadly informing shigellosis control in the critical demographic of children in LMICs. Here, we apply WGSA to Shigella isolates sampled during GEMS, representing 1,246 systematically collected isolates from across seven nations in sub-Saharan Africa and South Asia with some of the highest childhood mortality rates $(2,19)$. We found evidence of the potential benefit of genomic subtype-based targeting, characterised pathogen features that will complicate current vaccine approaches, and highlighted regional differences among Shigella diversity, as well as determinants of AMR, including convergent evolution toward resistance against currently recommended treatments. Our analysis of this unparalleled pathogen collection informs the control and prevention of shigellosis in those populations most vulnerable to disease.

\section{Results and Discussion}

\section{Regional diversity of Shigella spp. across LIMC}

To date, this is the largest representative dataset of Shigella genomes from LMICs $(n=1246)$, collected across seven sites from Asia, West Africa and East Africa, comprised of 806 S. flexneri, 305 S. sonnei, 75 S. boydii and 60 S. dysenteriae (Fig. 1A). To compare the genomic diversity of Shigella species, we determined the distributions of pairwise single-nucleotide polymorphism (SNP) distances and scaled 
the total detected SNPs against the length of the chromosome (in kbp) for each species (Fig. 1B). This revealed that $S$. boydii contained the greatest diversity (24.2 SNPs/kbp), followed by $S$. flexneri (19.5 $\mathrm{SNPs} / \mathrm{kbp}$ ) and $S$. dysenteriae (11.8 SNPs/kbp), with $S$. sonnei being >9.8-fold less diverse (1.2 SNPs/kbp) or >13.1-fold less diverse (0.9 SNPs/kbp) excluding two outliers (see below, Fig. 1B). This revealed that $S$. sonnei caused between 20 and 25 -fold more disease relative to genomic diversity than S. flexneri and either $S$. dysenteriae or S. boydii (Fig. 1B), indicating the value of vaccination against $S$. sonnei as a comparatively conserved target relative to disease burden. Examination of the gene repertoire revealed that this relative chromosomal diversity was consistent with the accessory genome variation among species (fig. S1).

Early global population structure studies revealed that each Shigella species is delineated into multiple WGSA subtypes (20-23). Specifically, S. flexneri is comprised of seven phylogroups (PGs) (20) and S. sonnei of five lineages (24). To describe the genomic epidemiology of the GEMS Shigella within existing frameworks we constructed species phylogenetic trees and integrated these with epidemiological metadata and publicly available genomes. The S. flexneri phylogeny revealed two distinct lineages separated by 34,000 SNPs; one comprising five previously described PGs (20) and a distant clade comprised largely of S. flexneri serotype 6 isolates (herein termed Sf6), contributing distinctly to the disease burden of each country (Fig. 2 and fig. S2). Phylogenetic analysis of S. sonnei revealed that all but two isolates belonged to the globally dominant multidrug resistant (MDR) Lineage III (21) (fig. S3). For $S$. boydii and $S$. dysenteriae, a total of four and two previously described phylogenetic clades $(23,25)$ were identified, respectively (fig. S4). Marked phylogenetic association of isolates with country of origin prompted an examination of species genomic diversity by region (East Africa, West Africa and Asia) and revealed that while $S$. flexneri diversity was comparable across regions, diversity varied by region for the remaining species (fig. S5). Specifically, S. sonnei was more genomically diverse in East Africa owing to the presence of two Lineage II isolates from Mozambique. For S. boydii, Asia contained greater diversity than African regions, owing to isolates belonging to additional clades. S. dysenteriae diversity was lower in West Africa relative to other regions by virtue of having only one circulating clade. These geographical differences highlight the importance of considering regional variations during vaccine development and that vaccine candidates should be evaluated across multiple regions.

\section{Genomic subgroups as an alternative targeting method}

To explore the utility of vaccination targeting genomic subtype (relative to targeting serotype) for S. flexneri, we determined the relative effect size of the dominant subtype on the epidemiological outcome 
of shigellosis (i.e., isolates derived from case patients rather than from controls, as defined in GEMS). The dominant genomic subtype was PG3, which comprised the majority (47\%, 378/806) of total isolates, as well as case $(50 \%, 341 / 687)$ isolates, with some regional variation (Fig. 2). This resulted in an increased odds of cases $(\mathrm{OR}=2.3,95 \% \mathrm{CI}=1.5-3.6, p=0.0001)$ for PG3 compared with other genomic subtypes (PGs and Sf6) (methods, table S3). The association of cases with the dominant serotype, S. flexneri serotype 2a (accounting for 29\% (234/806) of total isolates and 31\% (210/687) of case isolates) also resulted in an increased odds of cases $(\mathrm{OR}=1.9,95 \% \mathrm{CI}=1.7-3.2, p=0.0099)($ table $\mathrm{S} 3)$. But the higher prevalence and larger effect size of PG3 relative to serotype 2a on case status offers compelling evidence that targeting vaccination by phylogroup might offer broader coverage per licenced vaccine relative to, or in combination with, a serotype-specific approach.

\section{Diversity of S. flexneri relevant to serotype-targeted vaccines}

The development of serotype-targeted vaccines is complicated by the diversity and distribution of serotypes, which are heterogenous over time and place $(8,19,26,27)$. Furthermore, genetic determinants of O-antigen modification are often encoded on mobile genetic elements $(28,29)$ that can move horizontally among bacterial populations, causing the recognised, but poorly quantified phenomenon of serotype switching $(20,27,28)$, which may result in the rapid escape of infection induced immunity against homologous serotypes. For our analyses of serotype switching, we focused on S. flexneri owing to high disease burden and serotypic diversity. Phenotypic serotyping data were overlaid onto the phylogeny and revealed that while generally there was a strong association of genotype (i.e. PG/Sf6) with serotype (Fisher's exact test; $p<2.20 \mathrm{E}-16$ ), multiple serotypes were observed for each genotype (Fig. 3). The greatest serotype diversity was observed in PG3, comprised of seven distinct serotypes and two subserotypes. Correlation of serotypic diversity (number of serotypes) and genomic diversity (maximum pairwise SNP distance within genotype) revealed no evidence for an association, but a significant positive correlation of serotypic diversity with the number of isolates in each genotype was found (fig. S6), indicating that serotype diversity scales with prevalence.

To qualitatively and quantitatively determine serotype switching across S. flexneri, we examined the number of switches occurring within each genotype. A switching event was inferred when a serotype emerged (either as a singleton or monophyletic clade) that was distinct from the majority (>65\%) serotype within a genotype (Fig. 3 and fig. S7). PG6 was excluded from the analysis, as only three isolates from GEMS belonged to this genotype and a dominant serotype could not be inferred. Quantitatively, this 
revealed serotype switching was infrequent, with only 26 independent switches (3.3\% of isolates) identified across the five $S$. flexneri genotypes. Although the frequency of switching varied across the genotypes, statistical support for an association of serotype switching with genotype fell short of significance (Fisher's exact test; $p=0.09)$. Qualitatively, the majority (22/26) of switching resulted in a change of serotype, with few (4/26) resulting in a change of subserotype. Examination of O-antigen modification genes revealed that serotype switching was facilitated by changes in the composition of phage-encoded gtr and oac genes in the genomes, as well as point mutations in these genes (table S4). Our data also revealed that few (4/26) switching events resulted in more than two descendant isolates (fig. S7). This indicates that while natural immunity drives the fixation of relatively few serotype-switched variants in the short term, the potential pool of variants that could be driven to fixation by vaccine-induced selective pressure following a serotypetargeted vaccination program is much larger.

In order to estimate the likely timeframe over which serotype switching events might be expected to occur, we estimated the divergence time of the phylogenetic branch giving rise to each switching event. To streamline the analysis, we focused on two subclades of PG3, the most prevalent phylogroup, in which seven independent serotype switching events were detected (fig. S8). Based on the timeframes observed within our sample (spanning 4 years from 2007 to 2010), serotype switching was estimated to occur within an average of 348 days, ranging from 159 days (95\% highest posterior density [HPD]: 16 - 344) to 10206 days (28 years) (95\% HPD: 5494 - 15408) (table S5). Taken together, our data shows that although serotype-switching frequency is low, it can occur over relatively short timeframes and lead to serotype replacement such that non-vaccine serotypes could replace vaccine serotypes following a vaccination program, as has been observed for Streptococcus pneumoniae $(30,31)$. These elucidated serotype switching dynamics (i.e. switching occurring over short timeframes and quantitatively proportional to disease burden) highlights the value of a multivalent vaccine and geographically coordinated implementation of Shigella vaccination.

\section{Heterogeneity among Shigella vaccine protein antigens}

Conserved antigen-targeted vaccines can overcome some hurdles of serotype-targeted vaccines. Hence, we performed detailed examination of six protein antigens that are currently in development and have demonstrated protection in animal models (Table 1). First, we assessed the distribution of the candidates among GEMS Shigella isolates which revealed that the proportional presence of antigens varied across species and with genetic context. Specifically, genes encoded on the virulence plasmid (ipaB, ipaC, 
$i p a D$, icsP) were present in $>85 \%$ of genomes for each species with the exception of S. sonnei (fig. S9). The low proportion $(\leq 5 \%)$ of virulence plasmid encoded genes detected among $S$. sonnei was caused by a similarly low detection of the virulence plasmid among S. sonnei (6\%), which likely arose due to loss during sub-culture (32). In contrast, the chromosomally encoded ompA was present in $>98 \%$ of all isolates, while the sigA gene (carried on the chromosomally integrated SHI-1 pathogenicity island (17)) was present in $99 \%$ of $S$. sonnei genomes, but only $63 \%$ of S. flexneri genomes. Notably, among S. flexneri genomes, the sigA gene was exclusively found in PG3 and Sf6, and present in $>96 \%$ of isolates in each genotype) (fig. S2), indicating an appropriate distribution for targeting the two genotypes. Second, we assessed the antigens for amino acid variation and modelled the likely impact of detected variants, as antigen variation may also lead to vaccine escape, as demonstrated for the P1 variant of SARS-CoV2 $(33,34)$. We determined the distribution of pairwise amino acid (aa) sequence identities per antigen against $S$. flexneri vaccine strains for each species (methods). Overall, sequence identities were $>90 \%$ but varied with antigen (fig. S9). For example, OmpA was present in the highest proportion of genomes, but showed $\sim 5 \%$ sequence divergence, while SigA was present in fewer genomes, but exhibited little divergence $(<0.5 \%)$ among species. The least conserved sequence was IpaD, ranging from 3 to $7 \%$ divergence within species.

Not all antigenic variation will affect antibody binding, so we performed in silico analyses of the detected variants to assess whether they may compromise the antigens as vaccine targets. Again, we focused our analyses on S. flexneri owing to its high disease burden and the likely complication of serotype-based vaccination strategies for this species. We detected 121 variants across the six antigens, the majority (79\%) of which correlated with genotype (i.e. belonging to either PGs 1-5 or Sf6, fig. S11). We then determined if amino acid variants were located in immunogenic regions (i.e. epitope/peptide fragment) (fig. S10) and assessed their potential destabilization of protein structure through in silico protein modelling. For IpaB, IpaC and IpaD, the epitopes have been empirically determined $(35,36)$. The sequence and location of peptide fragments of SigA, IcsP and OmpA used in vaccine development are available $(37,38)$. Variants located within the immunogenic regions were identified for all antigens relative to PG3 reference sequences (methods, Fig. 4). Only 4 of 121 variants were predicted to be highly destabilising to protein structure, and these occurred in: OmpA (residue 89) at a periplasmic turn, SigA (residues 1233 and 1271) in adjacent extracellular turns in the translocator domain (fig. S12), and in IpaD (residue 247) within a beta-turn-beta motif flanking the intramolecular coiled-coil (Fig. 4). While it remains possible that these mutations could affect antigenicity through the disruption of folding or global stability, it is less likely than if they occurred in immunogenic regions. These results thus indicate that it is less likely that existing natural variation will compromise antigen-based vaccine candidates for Shigella compared with serotype-based vaccines. However, our approach is limited and the knowledge base incomplete. For example, there was no suitable 
template available for IpaC, and some epitopes were predicted to be in membrane regions which should be inaccessible to antibodies, indicating the need for more accurate publicly available protein structures to be developed for many of the vaccine antigen candidates.

\section{Region-specific details of antimicrobials as a stop gap}

Until a licensed vaccine is available, we must continue to treat shigellosis with supportive care and antimicrobials, for which the current WHO recommendation is the fluoroquinolone, ciprofloxacin (39). However, FQR Shigella is currently on the rise and spreading globally (40). To examine AMR prevalence among GEMS isolates for evaluating treatment recommendations, we screened for known genetic determinants (horizontally acquired genes and point mutations) conferring resistance or reduced susceptibility to antimicrobials. Although we used only minimal phenotypic data, phenotypic resistance and genotypic prediction correlate well in S. flexneri and S. sonnei $(41,42)$. Our analysis revealed that $95 \%$ (1189/1246) of isolates were multidrug resistant (MDR), carrying AMR determinants against three or more antimicrobial classes (Fig 5A). S. flexneri exhibited the greatest diversity of AMR determinants, with a total of 45 identified determinants across the population, comprising of 38 AMR genes and 7 point mutations (fig. S13 and table S1), and an extensive AMR genotype diversity of 72 unique resistance profiles (Fig. 5A and fig. S14). In contrast, S. sonnei exhibited the least diversity, with only 23 AMR determinants and 21 unique resistance profiles. An intermediate and comparable degree of AMR diversity was observed for both S. dysenteriae and S. boydii.

Overall, a high frequency of AMR genes conferring resistance against aminoglycoside, tetracycline, trimethoprim, and sulphonamide antimicrobials was observed, while resistance against other antimicrobial classes varied with region and species (Fig. 5B). The extended spectrum beta-lactamase gene blaCTX-M-15 was detected in a small (9/1246) percentage of isolates, and genes conferring resistance to macrolides and lincosamides were also infrequent (fig. S13), indicating that the recommended second-line treatments likely remain effective antimicrobials (43).

However, higher rates of resistance were found against the first-line treatment. FQR in Shigella can be conferred through the acquisition of FQR-genes or, more typically, by point mutations in the chromosomal Quinolone Resistance Determining Region (QRDR) within the DNA gyrase (gryA) and the topoisomerase IV ( parC) genes. Single and double QRDR mutations are known to confer reduced susceptibility to ciprofloxacin and are evolutionary intermediates on the path to resistance, conferred by 
triple mutations in this region $(41,44)$. Overall, FQR-genes were uncommon in S. flexneri $(4 \%, 33 / 806), S$. sonnei $(1 \%, 3 / 305)$ and $S$. dysenteriae $(7 \%, 4 / 60)$, but were present in 32\% (24/75) of S. boydii. QRDR mutations were identified in all species (fig. S13), but were more common among S. sonnei $(65 \%, 199 / 305)$ and S. flexneri $(54 \%, 435 / 806)$ than compared with S. boydii $(15 \%, 11 / 75)$ and S. dysenteriae $(30 \%, 18 / 60)$. Among these, triple QRDR mutations were identified in 13\% (106/806) of S. flexneri and 14\% (44/305) of S. sonnei. Analysis of the QRDR mutants across the phylogenies indicate marked convergent evolution toward resistance across the genus. Specifically, all triple QRDR mutant S. sonnei belonged to one monophyletic subtype (previously described as globally emerging from Southeast Asia (45)), while three distinct triple QRDR mutational profiles were found across three polyphyletic S. flexneri genotypes (Fig. 5C). Thus, the polyphyletic distribution of single, double, and triple QRDR mutants indicates continued convergent evolution of lineages with reduced susceptibility or resistant to FQR.

We then stratified the dataset by geographic region which revealed that $\mathrm{FQR}$ were largely associated with isolates from Asia where fluoroquinolones are more frequently used compared to African sites (Fig. 5B) (46), which is consistent with trends observed in atypical enteropathogenic Escherichia coli isolated from GEMS (46). Our analyses thus suggest that for the period of GEMS trial (2007 - 2011), 17\% (150/881) of Shigella isolates from Asia were resistant and 58\% (508/881) had reduced susceptibility to the WHO recommended antimicrobial. The high level of reduced susceptibility together with marked convergent evolution toward resistance suggests that management of shigellosis with fluroquinolones at these sites may soon be ineffective and regional antimicrobial treatment guidelines may require updating. These results indicate the value of AMR and microbiological surveillance in LMICs and the control and management of shigellosis will be improved by initiatives such as the Africa Pathogen Genomics Initiative (47) and the WHO Global Antimicrobial Resistance Surveillance System (48).

\section{Conclusions}

Pathogen genomics is a powerful tool that has a wide range of applications to help combat infectious diseases. Here, we have applied this tool to an unparalleled systematically collected Shigella dataset to characterise the relevant population diversity of this pathogen across LMICs in a pre-vaccine era. Our results revealed that current antimicrobial treatment guidelines for shigellosis should be updated, and that improved surveillance will be essential to guide antimicrobial stewardship. This study has also highlighted the urgent need to continue the development of Shigella vaccines for children in endemic areas. The genomic diversity in Shigella presents a major hurdle in controlling the disease and we have demonstrated the anticipated pitfalls of current vaccination approaches, emphasising the importance of 
considering the local and global diversity of the pathogens in vaccine design and implementation. Although our results are focused on shigellosis, our approach is translatable to other bacterial pathogens which is particularly relevant as we enter the era of vaccines for AMR.

\section{Materials and Methods}

\section{Dataset, bacterial isolates and sequencing}

A total of 1,264 Shigella isolates from GEMS were under investigation in this study $(2,3)$. All isolates were derived from stool samples/rectal swabs: their identification, confirmation and isolation have been described previously (19). A total of 1,344 isolates were sequenced at the Earlham institute, with genomic DNA extraction, sequencing library construction and whole genome sequencing carried out according to the Low Input Transposase Enabled (LITE) pipeline described by Perez-Sepulveda et al (49). Among these, 225 isolates failed QC with a mean sample depth of coverage <10x and an assembly size of <4MB and were re-sequenced. For these isolates, genomic DNA was re-extracted at the University of Maryland School of Medicine (Baltimore, Maryland) from cultures grown in Lysogeny Broth overnight. DNA was extracted in 96-well format from $100 \mu \mathrm{L}$ of sample using the MagAttract PowerMicrobiome DNA/RNA Kit (Qiagen, Hilden, Germany) automated on a Hamilton Microlab STAR robotic platform. Bead disruption was conducted on a TissueLyser II ( $20 \mathrm{~Hz}$ for $20 \mathrm{~min}$ ) instrument in a 96 deep well plate in the presence of 200 $\mu \mathrm{L}$ phenol/chloroform. Genomic DNA was eluted in $90 \mu \mathrm{l}$ water after magnetic bead clean up and the resulting genomic DNA was quantified by Pico Green. The genomic DNA was shipped to the Centre for Genomic Research (University of Liverpool) for whole genome sequencing. Sequencing library was constructed using NEBNext ${ }^{\circledR}$ Ultra ${ }^{\mathrm{TM}}$ II FS DNA Library Prep Kit for Illumina and sequenced on the Illumina ${ }^{\circledR}$ NovaSeq 6000 platform, generating 150bp paired-end reads.

An additional 125 publicly available Shigella and E. coli reference genomes were included in the analyses. Details of GEMS and reference genomes analysed in this study are listed in table S1 and table S2, respectively. 
Adaptors and low-quality bases were trimmed with Trimmomatic v0.38 (50), reads qualities were assessed using FastQC v0.11.6 (https://www.bioinformatics.babraham.ac.uk/projects/fastqc/) and MultiQC v1.7 (51). Filtered reads were mapped against Shigella reference genomes with BWA mem v0.7.17 (52) using default parameters. S. flexneri, S. sonnei, S. boydii and S. dysenteriae sequencing reads were mapped against reference genomes from Sf2a strain 301 (accession NC_004337), Ss046 (accession NC_007384), Sb strain CDC 3083-94 (accession NC_010658) and Sd197 (accession NC_007606), respectively. Mappings were filtered and sorted using the SAMtools suite v1.9-47 (53), and optical duplicate reads were marked using Picard v2.21.1-SNAPSHOT MarkDuplicates (http://broadinstitute.github.io/picard/). QualiMap v2.2.2 (54) was used to evaluate mapping qualities and estimate mean sample depth of coverage. SAMtools v1.9-47 mpileup and bcftools v1.9-80 (53). Low quality SNPs were filtered if mapping quality $<60$, Phred-scaled quality score $<30$ and read depth $<4$.

\section{Phylogenetic reconstruction and inference of genomic diversity}

Filtered SNP variants were used to generate a reference-based pseudogenome for each sample, where regions with depth of coverage $>4 \mathrm{x}$ were masked in the pseudogenome. Additionally, regions containing phage (identified using PHASTER (55)) and insertion sequences were identified from the reference genomes, and co-ordinates were used to mask these sites on the pseudogenomes using BEDTools v2.28.0 maskfasta (56). For each species, chromosome sequences from the masked pseudogenomes were extracted and concatenated. Gubbins v2.3.4 (57) was used to remove regions of recombination and invariant sites from the concatenated pseudogenomes. This generated a chromosomal SNP alignment length of

$33278,251 \mathrm{bp}$ for $S$. flexneri ( $n=806), 5,081 \mathrm{bp}$ for $S$. sonnei $(n=305), 98,842 \mathrm{bp}$ for $S$. boydii $(n=75)$ and 45,031

333 bp for $S$. dysenteriae $(n=60)$. Maximum-likelihood phylogenetic reconstruction was performed independently for each species and inferred with IQ-TREE v2.0-rc2 (58) using the FreeRate nucleotide substitution, invariable site and ascertainment bias correction model, with 1000 bootstrap replicates. In order to contextualise GEMS isolates within the established genomic subtypes and to infer the most appropriate root for each species tree, phylogenetic trees were reconstructed including publicly available reference genomes of isolates from previously defined lineages/phylogroups/clades and E. coli isolates 
IAI1-117 (accession SRR2169557) as an outgroup, respectively. Phylogenetic tree for S. sonnei was midpoint rooted. Visualizations were performed using interactive Tree of Life (iTOL) v6.1.1 (59).

To measure the extent of shigella genomic diversity among GEMS population, pairwise SNP distance was determined from the alignment of core genome SNPs identified outside regions of recombination using snp-dists v0.7.0 (https://github.com/tseemann/snp-dists). For each species, the genomic diversity, measured by SNPs per kbp, was determined by dividing the core genome SNP alignment length by the core genome size (S. flexneri 4,015,307 bp, S. sonnei 4,177,070 bp, S. boydii 4,088,693 bp and $S$. dysenteriae 3,821,602 bp). Scaling the proportion of disease burden attributable by the genome diversity of each species, the percentage of species contribution to GEMS shigellosis disease burden was divided by the number of SNPs per kbp.

\section{Serotype switching time frame inference}

To estimate the likely time frame of serotype switching, we performed temporal phylogenetic reconstruction in order to infer the time of divergence along branches exhibiting serotype switching. We streamlined the analysis and focused on isolates belonging to two subclades of $S$. flexneri PG3. First, for each of the two subclades ( $n=99$ and $n=45$ ), a maximum-likelihood phylogeny was reconstructed based on genome multiple sequence alignments (described above). Then, TempEst v1.5.3 (60) was used determine if there is sufficient temporal signal in the data by inferring linear relationship between root-to-tip distances of the phylogenetic branches with the year of sample isolation. Data from both subclades revealed positive correlation between sampling time and phylogenetic root-to-tip divergence, with $\mathrm{R}^{2}$ of 0.186 and 0.111 (fig. S16). Once temporal signals within each of the two datasets were confirmed, core genome SNP alignments of length $559 \mathrm{bp}$ and 1,244 bp were analysed independently using BEAST2 v2.6.1 (61). The parameters were as follows: dates specified as days, bModelTest (62) implemented in BEAST2 was used to infer the most appropriate substitution model, a relaxed log normal clock rate with a coalescent Bayesian skyline model for population growth. A total of five independent chains were performed, each with chain length of $250,000,000$, logging every 1,000 and accounting for invariant sites. Convergence of each run was visually assessed with Tracer v1.7.1 (63), with all parameter effective sampling sizes $\geq 200$. Tree files were sampled and combined using LogCombiner v2.6.1, the combined files were then summarised using TreeAnnotator v2.6.0 with $10 \%$ burn-in to generate Maximum Clade Credibility tree (64). Divergence time was inferred 
371 by reading the branch length from the most recent common ancestor to the first sampled isolate that

372 serotype-switched.

\section{Genome assembly and annotation}

375

376

377

378

379

380

381

382

383

384

385

386

387

388

389

390

391

392

393

Draft genome sequences were assembled using Unicycler v0.4.7 (65) with -min_fasta_length set to 200. QUAST v5.0.2 (66) was used to assess the qualities of the assemblies. Assemblies with total assembly length outside the range of $<4 \mathrm{Mbp}$ and $>6.4 \mathrm{Mbp}$ were removed. Resulting in an average length of 4,275,508 bp (range: 4 4,004,109 - 4,538,734 bp) for S. flexneri, 4,264,097 bp (range: 4,008,630 4,779,279 bp) for S. sonnei, 4,227,671 bp (range: 4,000,714 - 4,689,815 bp) for S. boydii and 4,297,921 bp (range: 4,040,642 - 4,659,860 bp) for S. dysenteriae. An average N50 value of 29,804 bp (range: 6,810 $34,658 \mathrm{bp}$ ) was generated for S. flexneri, 23,961 bp (range: 11,547 - 30,008 bp) for S. sonnei, 20,835 bp (range: 15,323 - 40,119 bp) for S. boydii and 22,137 bp (range: 14,090 - 31,358 bp) for S. dysenteriae. Draft genomes were annotated using Prokka v1.13.3 (67).

\section{Pangenome analysis}

The pangenome of each species was defined using Roary v3.12.0 (68) without splitting paralogues. The pangenome accumulation curves were generated separately for each species using the specaccum function from Vegan v2.5-7 (https://github.com/vegandevs/vegan/), with 100 permutations and random subsampling. Inspections of the variable gene content showed that all four species had open pangenomes, implying that the number of unique gene count increases with the addition of newly sequenced genomes.

\section{Shigella flexneri molecular serotyping}

Shigella serotype data was provided by collaborators at the University of Maryland School of Medicine (Baltimore, Maryland), serotyping was performed as previously describe (19). In silico serotyping of S. flexneri genomes was performed using ShigaTyper v1.0.6 (69) which detects the presence of serotypedetermining genetic elements from sequencing reads to predict serotype. ShigaTyper predictions were $84 \%$ 
concordant to the serotype data provided. SRST2 v2 (70) was used to detect mutations within serotypedetermining genetic elements, run against ShigaTyper sequence database with default parameters.

\section{Protein antigen screening}

To determine the presence of antigen vaccine candidates among GEMS Shigella isolates, genes of the antigen vaccine candidates was screened against draft genome assemblies using screen_assembly (17) with a threshold of $\geq 80 \%$ identity and $\geq 70 \%$ coverage to the reference sequence. Reference sequences for $i p a B$, ipaC, ipaD and icsP were derived from S. flexneri 5a strain M90T (accession GCA_004799585) and ompA and sigA was derived from S. flexneri 2a strain 2457T (accession NC_004741), both strains are commonly used in the laboratory for vaccine development. Antigen sequence variations were determined by examining the BLASTp (71) percentage identity against relevant query reference sequence. Allelic variations of antigen vaccine candidates among S. flexneri population were identified manually by visualising amino acid sequence alignments using AliView v1.26 (72).

\section{Protein antigen modelling}

In order to assess the effect of point mutations on protein stability and vaccine escape, six antigen candidates from $S$. flexneri PG3 were modelled: OmpA, SigA, IcsP, IpaB, IpaC and IpaD (Table 1). PG3 was selected as it is the most prevalent phylogroup and is therefore the target of current vaccine development. To model the antigen targets, we first searched for a suitable template using HHPred $(73,74)$. Five of the six proteins (OmpA, SigA, IcsP, IpaB and IpaD) had suitable homologues available. To improve the performance of the comparative modelling, the signal peptides for OmpA, SigA and IcsP were removed and OmpA, SigA and IpaB were modelled in two parts to make use of optimal templates. RosettaCM (75) was used to generate 200 models for each of the five proteins using the single best available template. For IpaC, where no suitable templates were available, trRosetta (76) was used to create five de novo predicted models. The best model for each antigen candidate was selected using QMEAN's average local score. QMEANbrane $(77,78)$ was used for suitable membrane proteins (IpaB, IpaC \& IpaD), otherwise QMEANDisCo (77) was used (table 6). Full details of the modelling and ranking are shown in table 7. The 
429

effect of point mutations on the stability of the antigen candidates was assessed using PremPS, and the default criterion of $\left(\Delta \Delta \mathrm{G}>1 \mathrm{kcal} \mathrm{mol}^{-1}\right)$ used to defining highly destabilising mutations (79).

\section{Detection of AMR genetic determinants and AMR testing}

To detect the presence of known genetic determinants for AMR, AMRFinderPlus v3.9.3 (80) was used to screen draft genome assemblies against the AMRFinderPlus database, which is derived from the Pathogen Detection Reference Gene Catalog (https://www.ncbi.nlm.nih.gov/pathogens/). AMRFinderPlus was performed with the organism-specific option for Escherichia, to screen for both point mutations and genes, and filter out uninformative genes that were nearly universal in a group. Output was then filtered to remove genetic determinants identified with $\leq 80 \%$ coverage and $\leq 90 \%$ identity. The presence of $S$. sonnei virulence plasmid was confirmed using short-read mapping using BWA mem (as described above) against the reference virulence plasmid from Ss046 (GenBank accession CP000039.1). Presence of the plasmid was defined by mapping of $>60 \%$ breadth of coverage across the reference. Visualisations of AMR resistance profiles were performed with UpSetR v2.1.3 (81). Four S. flexneri isolates with triple QRDR mutations were phenotypically tested for ciprofloxacin resistance using the Kirby-Bauer standardized disk diffusion method (82).

\section{Statistical analyses}

The strength of association between $S$. flexneri genomic subtype and serotype with the occurrence of case outcome was calculated using MedCalc's odds ratio calculator v20 (https://www.medcalc.org/calc/odds ratio.php) to report the odds ratio, 95\% confidence interval and statistical association. Association of genomic subtype with serotype and serotype switching was tested using Fisher's exact test. Linear regression analysis was used to determine the correlation between serotype diversity to various properties of genomic subtype. Both analyses were performed using R v4.0.3. 


\section{Acknowledgements}

We acknowledge and thank members of Baker group and Lab $\mathrm{H}$ at the University of Liverpool, and Rodrigo Bacigalupe at KU Leuven for invaluable discussions. We also thank Jay Hinton and Blanca Perez a technology directorate voucher from the University of Liverpool, by the National Institute of Allergy and Infectious Diseases, National Institutes of Health, Department of Health and Human Services under grant number U19AI110820, and by both a Global Challenges Research Fund (GCRF) data and resources grant BBS/OS/GC/000009D and the BBSRC Core Capability Grant to the Earlham Institute BB/CCG1720/1. Next-generation sequencing and library construction were delivered via the BBSRC National Capability in

471 Genomics and Single Cell (BB/CCG1720/1) at Earlham Institute, by members of the Genomics Pipelines

472 Group. RJB is funded by a Biotechnology and Biological Sciences Research Council Doctoral Training

473 Partnership studentship (BB/M011186/1). KSB is supported by a Wellcome Trust Clinical Research Career

474 Development Award (106690/A/14/Z) and an Academy of Medical Sciences Springboard award 475 (SBF002/1114), and is affiliated to the National Institute for Health Research Health Protection Research 476 Unit (NIHR HPRU) in Gastrointestinal Infections at University of Liverpool in partnership with Public 477 Health England (PHE) and collaboration with University of Warwick. The views expressed are those of the author(s) and not necessarily those of the NHS, the NIHR, the Department of Health and Social Care or

\section{Author contributions}

R.J.B performed majority of the data analysis and interpretation of the results under the scientific guidance of K.S.B. A.J.S and D.J.R performed in silico protein antigens modelling and prediction of the impacts of amino acid substitutions on protein stability. C.V.P supported Bayesian Evolutionary Analysis by Sampling Trees. S.M.T. prepared and provided GEMS Shigella isolates and metadata. DR contributed to sample preparation. R.J.B and K.S.B drafted the manuscript. All authors contributed to editing of the manuscript. 
bioRxiv preprint doi: https://doi.org/10.1101/2021.06.09.447709; this version posted June 10, 2021. The copyright holder for this preprint (which was not certified by peer review) is the author/funder, who has granted bioRxiv a license to display the preprint in perpetuity. It is made available under aCC-BY 4.0 International license.

A

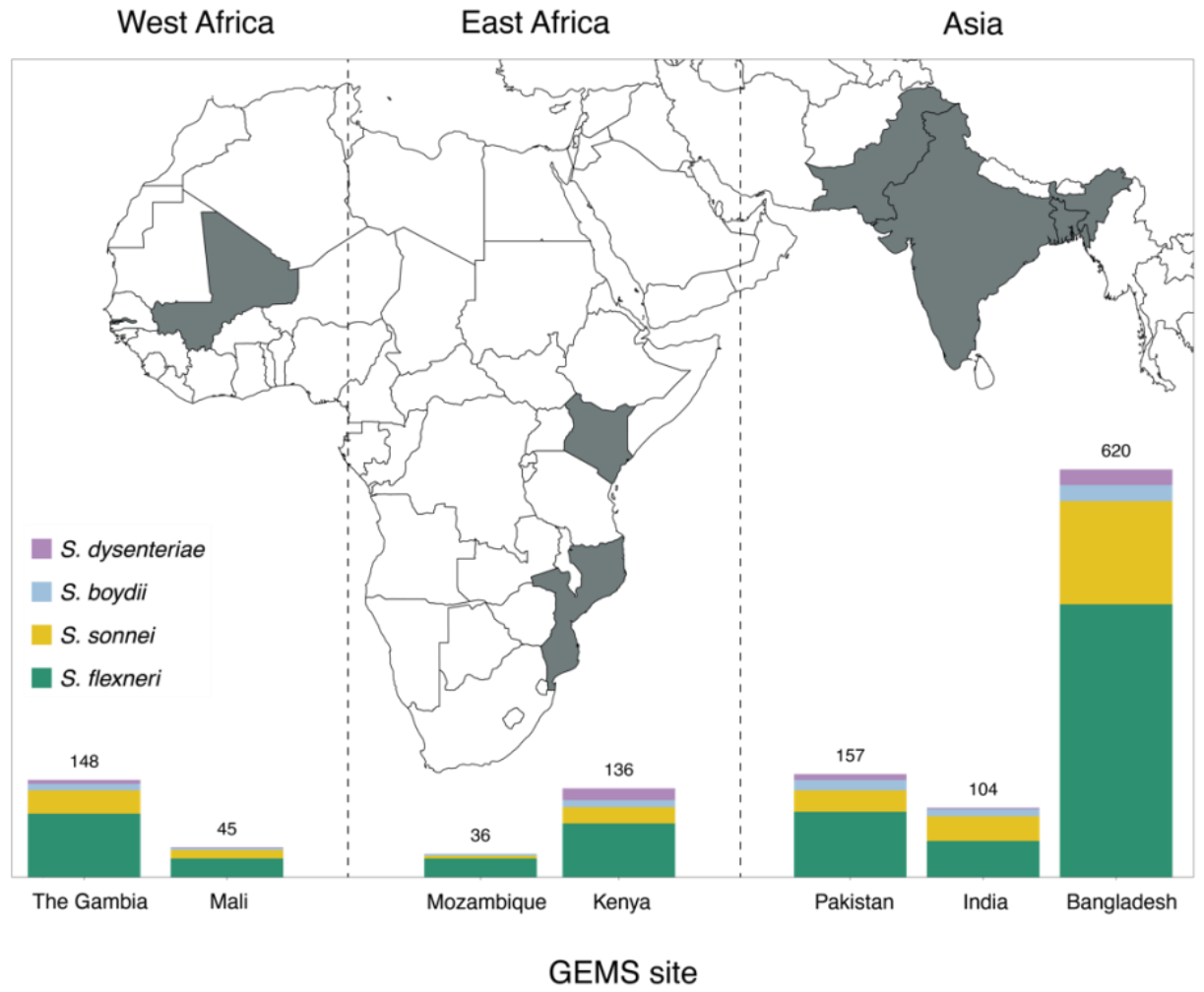

B

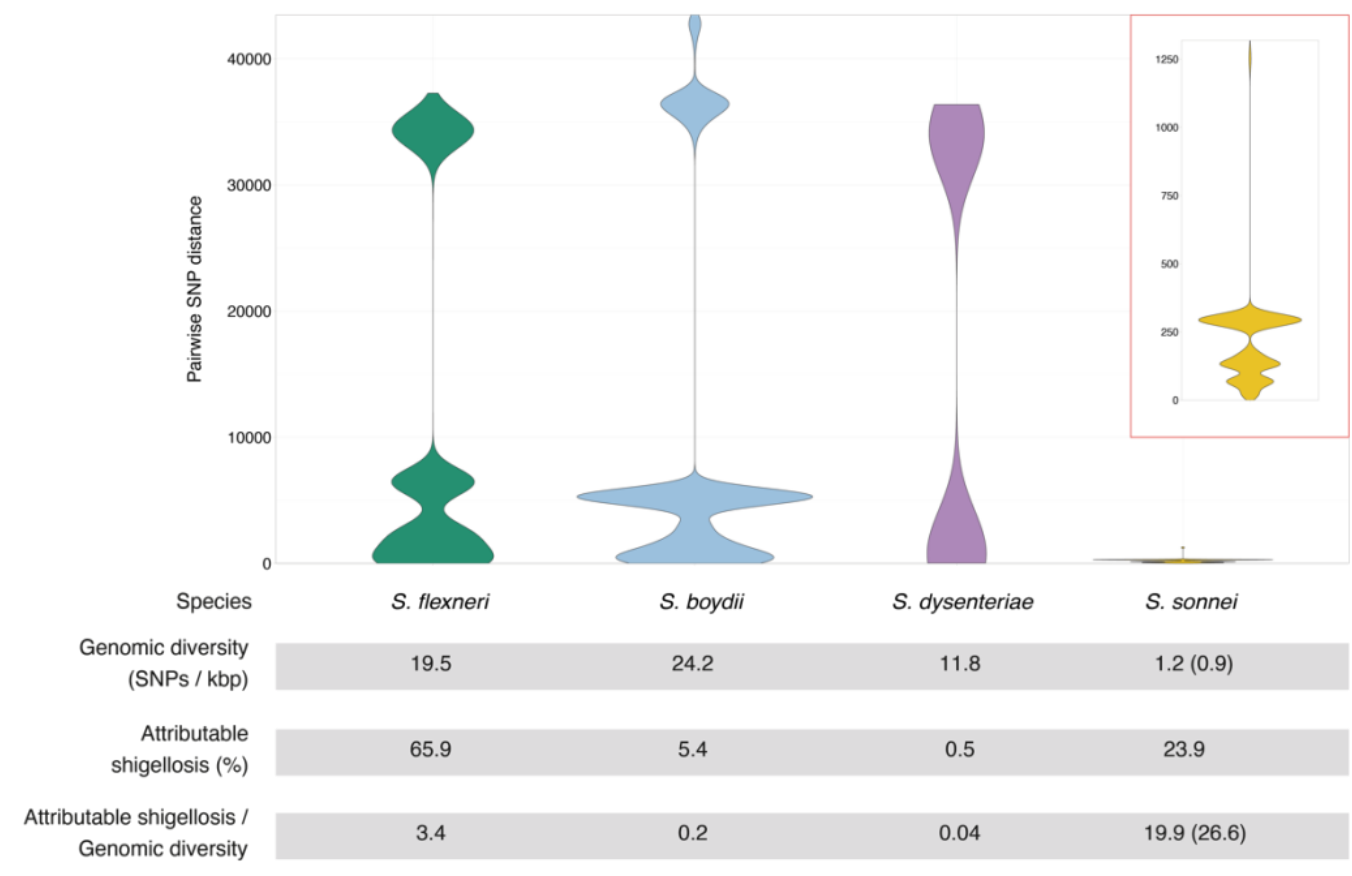


489 Fig. 1. The diversity of Shigella spp. across seven LMIC. (A) Stacked bar graphs illustrate the number 490 of isolates from each Shigella spp. sequenced from GEMS and used in the current study, grouped by study 491 sites. (B) Pairwise genomic distances (in SNPs) among Shigella isolates within subgroups are shown as 492 violin plots. A magnified plot for $S$. sonnei is displayed inside the red box. The table below the plots 493 demonstrates for each species the genomic diversity (as measure by total number of SNPs per kbp 494 [methods]), the contribution to GEMS shigellosis burden and the shigellosis burden relative to genomic 495 diversity. For S. sonnei, the genomic diversity and shigellosis burden relative to genomic diversity that was 496 calculated excluding the two outliers are shown in bracket. 


\section{S. flexneri genomic subtype}

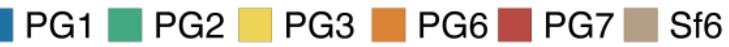
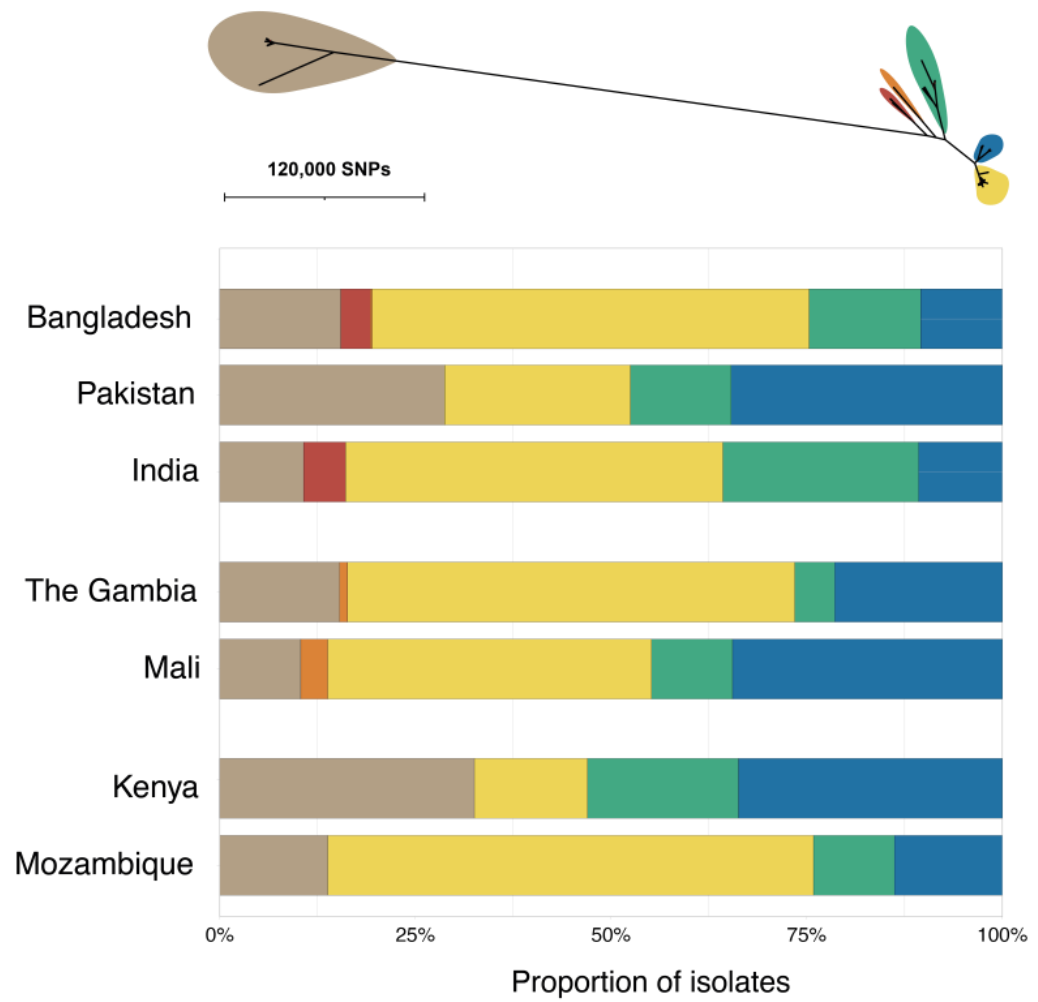

498 Fig. 2. The diversity of S. flexneri genomic subtypes across seven GEMS study sites. An unrooted ML phylogenetic tree of $S$. flexneri genomes identified six distinct genomic subtypes, each highlighted in a different colour according to the inlaid key displayed above the tree. The bar plot below the tree demonstrates the relative frequencies of the subtypes at each study site. 


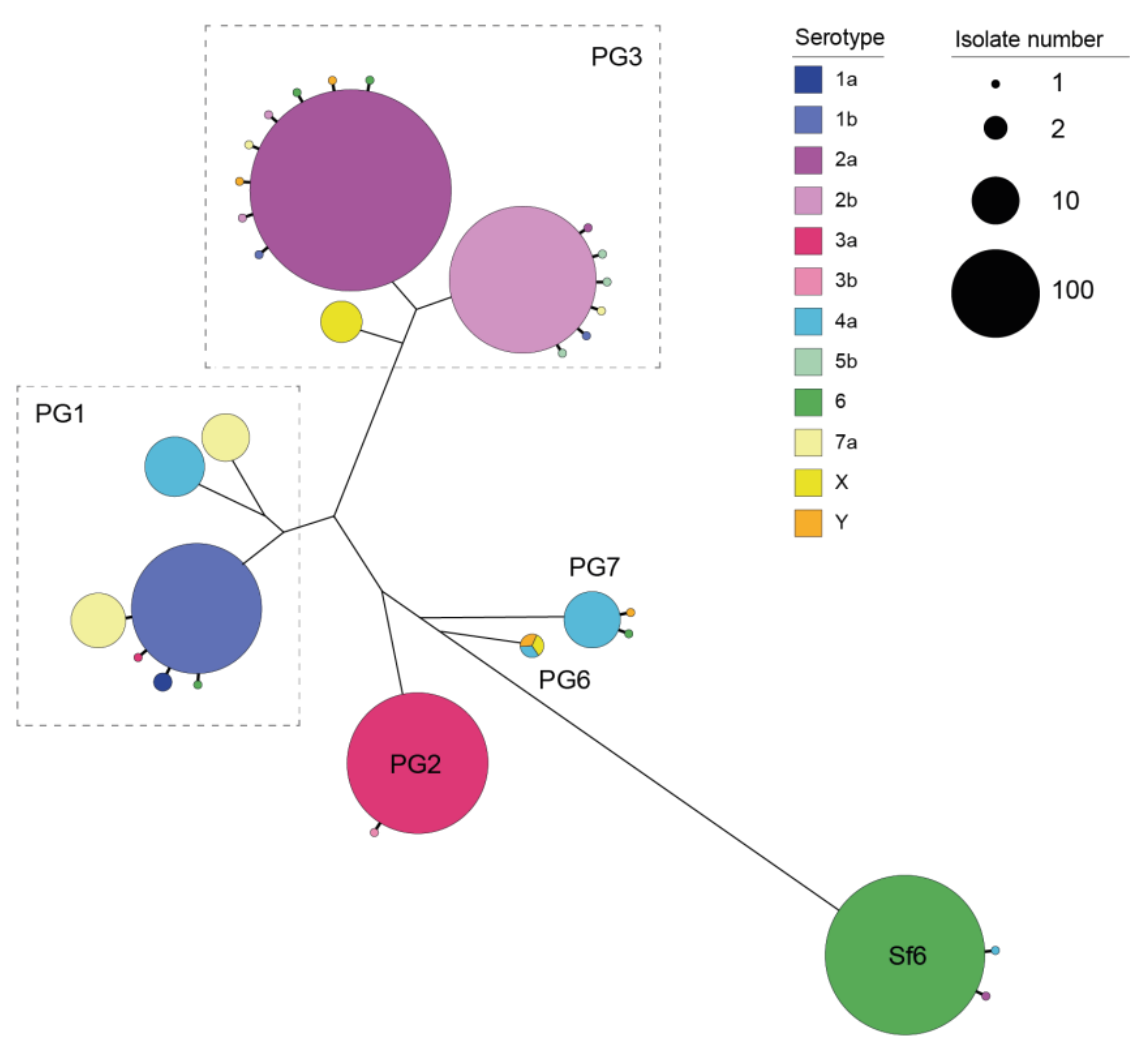
phylogenetic tree is shown with the five phylogroups (PG1-PG7) and Sf6 labeled accordingly. For each genomic subtype, monophyletic clusters of the dominant serotype are shown collapsed into bubbles coloured according to the inlaid key. Single isolates or groups of isolates within a subtype of an alternative serotype are represented by further branches, indicating a single serotype switch. The number of isolates within a single cluster is represented through bubble size. 
bioRxiv preprint doi: https://doi org/10.1101/2021.06.09.447709; this version posted June 10, 2021. The copyright holder for this preprint (which was not certified by peer review) is the author/funder, who has granted bioRxiv a license to display the preprint in perpetuity. It is made available under aCC-BY 4.0 International license.
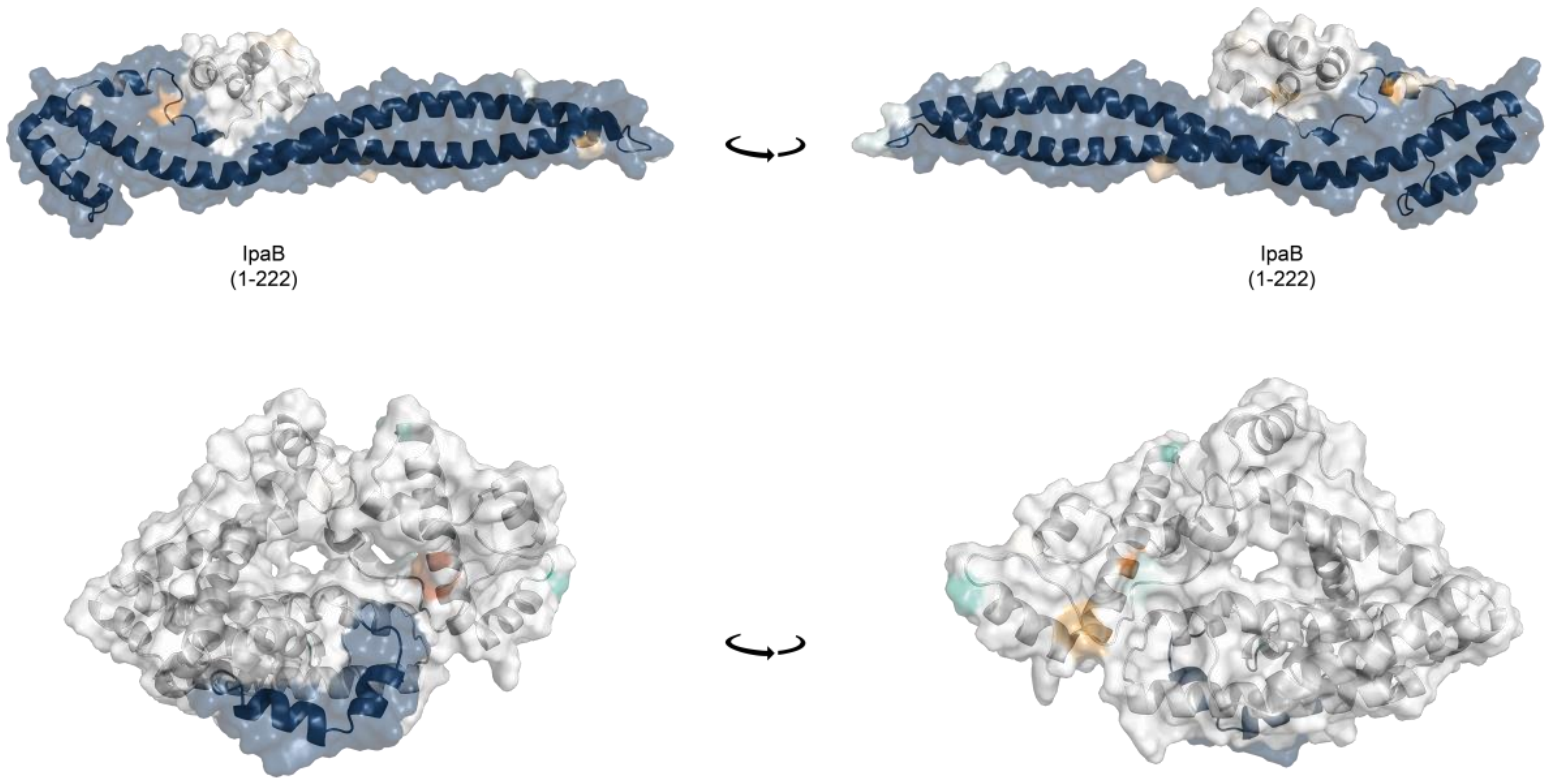

IpaB
$(223-553)$

$\mathrm{IpaB}$
$(223-553)$

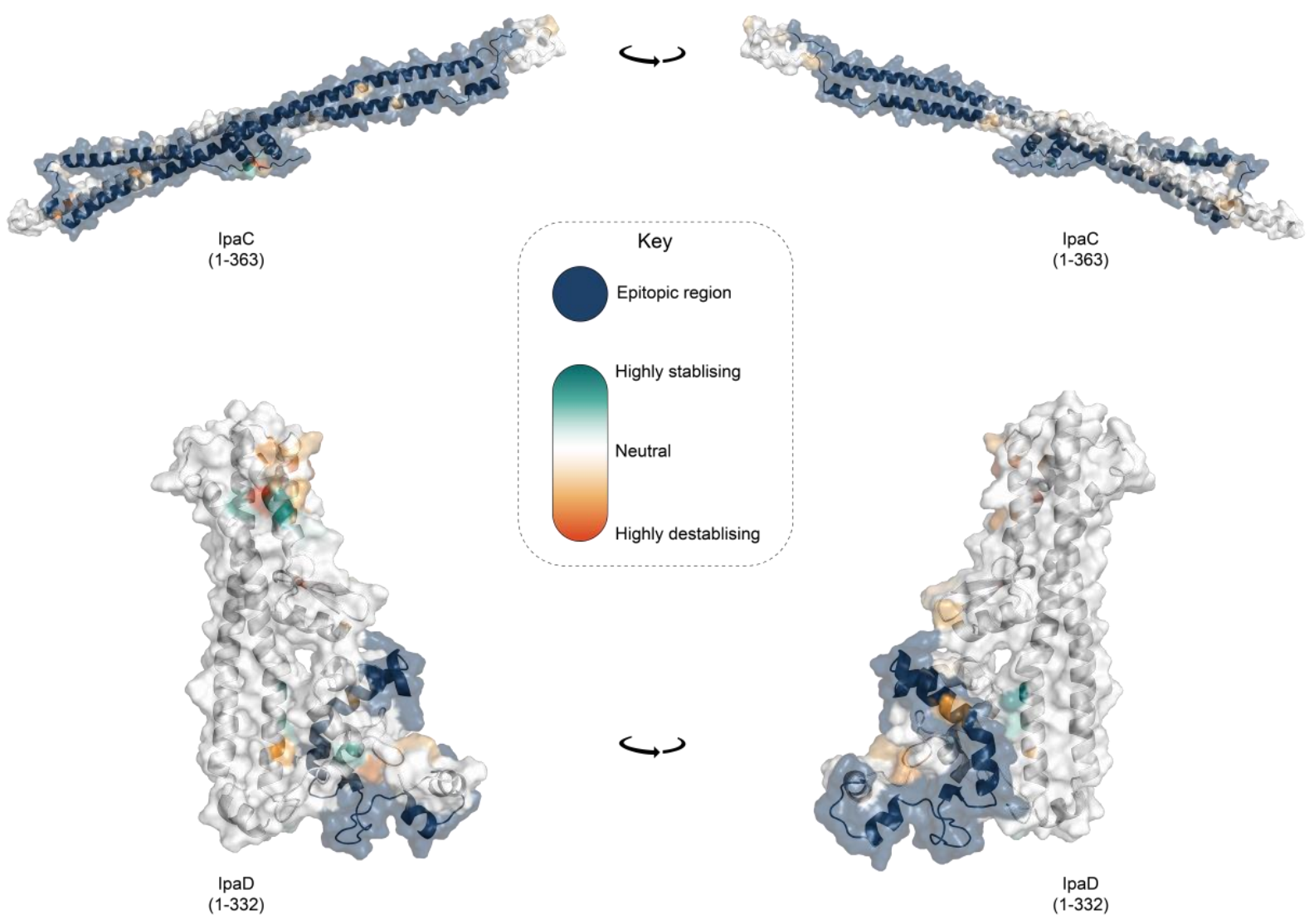


509 Fig. 4. Visualization of mutations and its predicted effect on modeled IpaB, IpaC and IpaD protein

510 antigens. Visualisation of mutations on modelled proteins IpaB, IpaC and IpaD. The protein residue ranges

511 modelled are shown in brackets. Blue region represents empirically determined epitopes. Mutations

512 identified within the proteins are coloured using the scale shown in the inlaid key, where highly

513 destabilising mutations are dark orange and highly stabilising mutations are dark green. 
bioRxiv preprint doi: https://doi.org/10.1101/2021.06.09.447709; this version posted June 10,2021. The copyright holder for this preprint (which was not certified by peer review) is the author/funder, who has granted bioRxiv a license to display the preprint in perpetuity. It is made available under aCC-BY 4.0 International license.

A

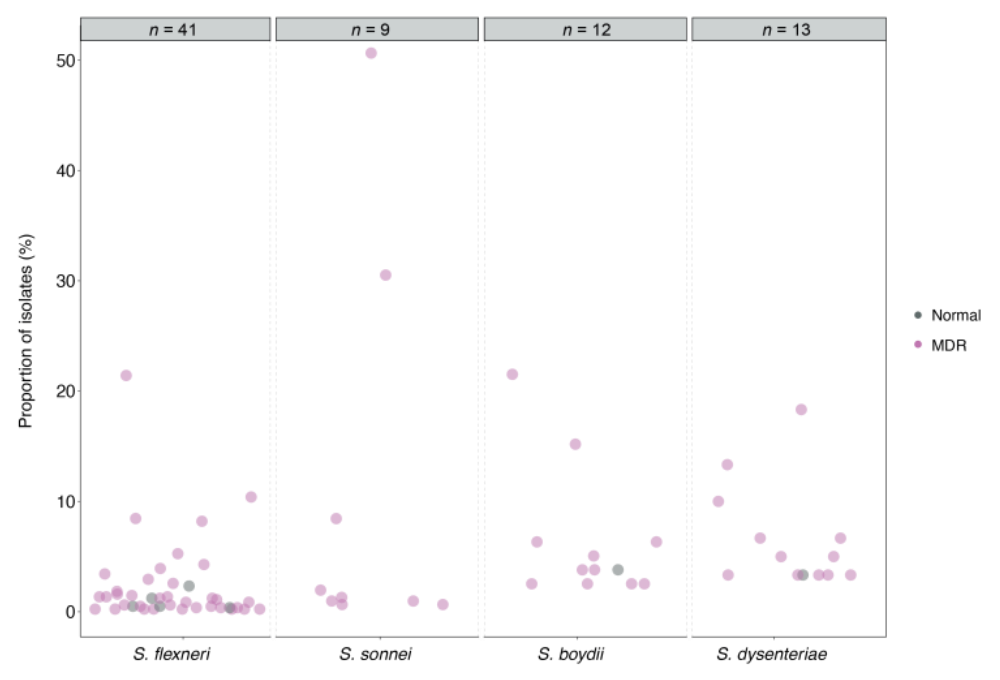

B

S. flexneri

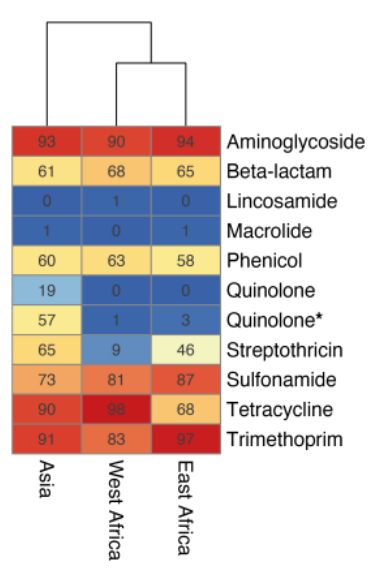

C

\section{S. sonnei}
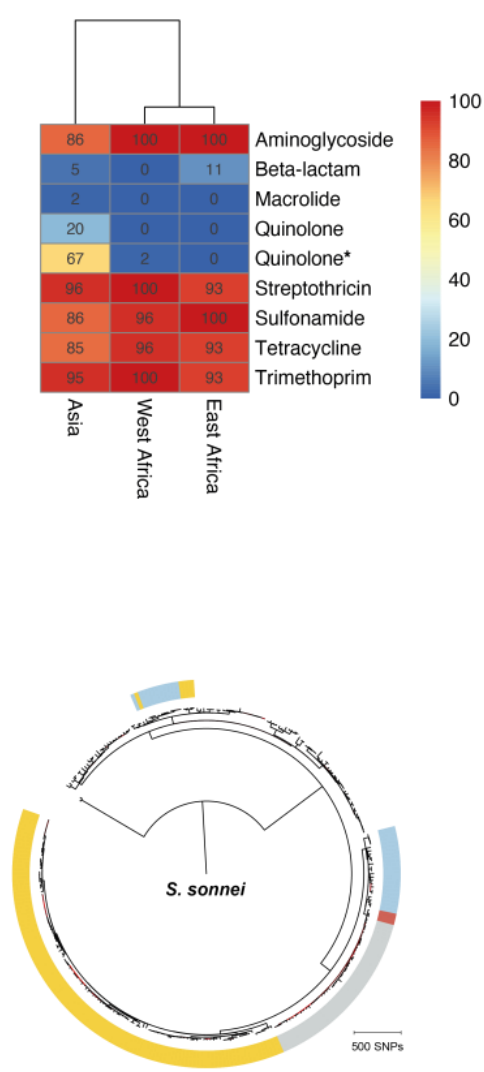

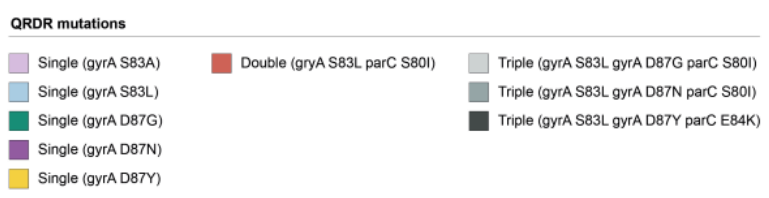


515 Fig. 5. AMR genotypic profile diversity and convergent evolution of ciprofloxacin resistance. (A) Frequencies of AMR genotypic profiles among Shigella spp. Each point in the scatterplot represents a

517 unique AMR genotype profile: the proportion of isolates with a particular profile is displayed along the y518 axis. Profiles identified in only a single isolate are not displayed. MDR genotypic profile conferring resistance or reduced suppressibility to three or more drug classes are highlighted in pink, and normal AMR genotype profile conferring resistance or reduced suppressibility in fewer than three drug classes are in grey. Numbers displayed above the plot represents the number of AMR genotype profiles plotted for each species. (B) Detection of known AMR genetic determinants associated with drug class grouped by country. Each cell in the heatmap represents the percentage of isolates from a region containing genetic determinants associated with resistance to a drug class. Genetic determinant conferring reduced susceptibility to quinolone is indicated with an asterisk. (C) The genetic convergent evolution of ciprofloxacin resistance in S. flexneri and S. sonnei. The presence of multiple monophyletic clades of QRDR mutations (single, double,

527 or triple according to the inlaid key) conferring reduced susceptibility or resistance to ciprofloxacin is shown in the outer ring. B and C for S. boydii and S. dysenteriae are shown elsewhere (fig. S15). 
529 Table 1. Shigella antigen vaccine candidates examined in the current study.

\begin{tabular}{|l|l|l|l|}
\hline $\begin{array}{l}\text { Vaccine } \\
\text { candidate }\end{array}$ & $\begin{array}{l}\text { Development } \\
\text { stage }\end{array}$ & Location & Reference \\
\hline IcsP $($ OmpP) & Preclinical & Virulence plasmid & Czerkinsky and Kim (37) \\
\hline SigA & Preclinical & $\begin{array}{l}\text { Chromosome } \\
\text { (pathogenicity island) }\end{array}$ & Czerkinsky and Kim (37) \\
\cline { 1 - 1 } IpaB & Phase I & Virulence plasmid & $\begin{array}{l}\text { Martinez-Beccera } \\
\text { Riddle et al (83); Tribble et al } \\
(84)\end{array}$ \\
\cline { 1 - 1 } IpaC & PpaD & Chromosome & Pore et al $(85)$ \\
\hline OmpA & & & \\
\hline
\end{tabular}


bioRxiv preprint doi: https://doi.org/10.1101/2021.06.09.447709; this version posted June 10, 2021. The copyright holder for this preprint (which was not certified by peer review) is the author/funder, who has granted bioRxiv a license to display the preprint in perpetuity. It is made available under aCC-BY 4.0 International license.

A

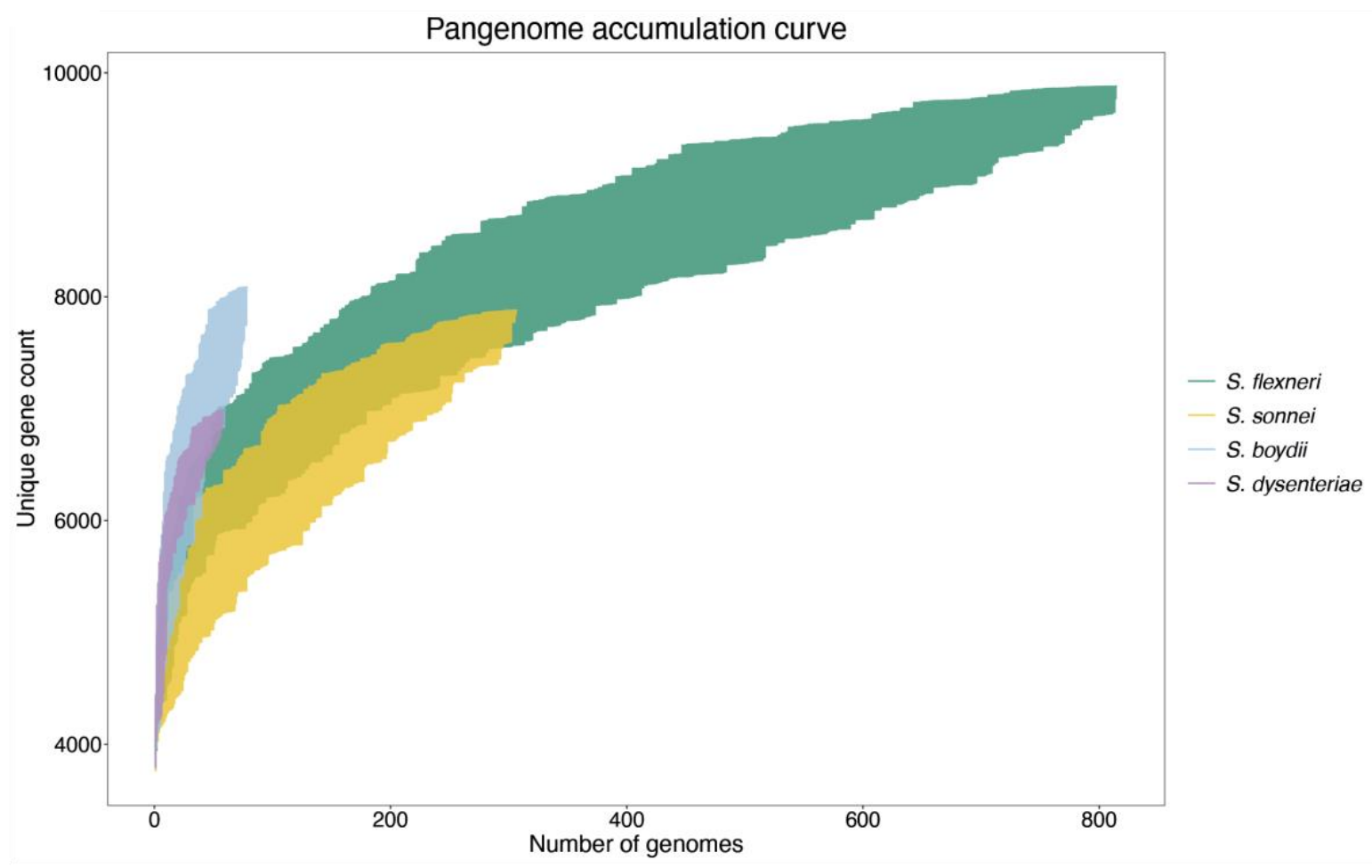

B

Pangenome accumulation curve

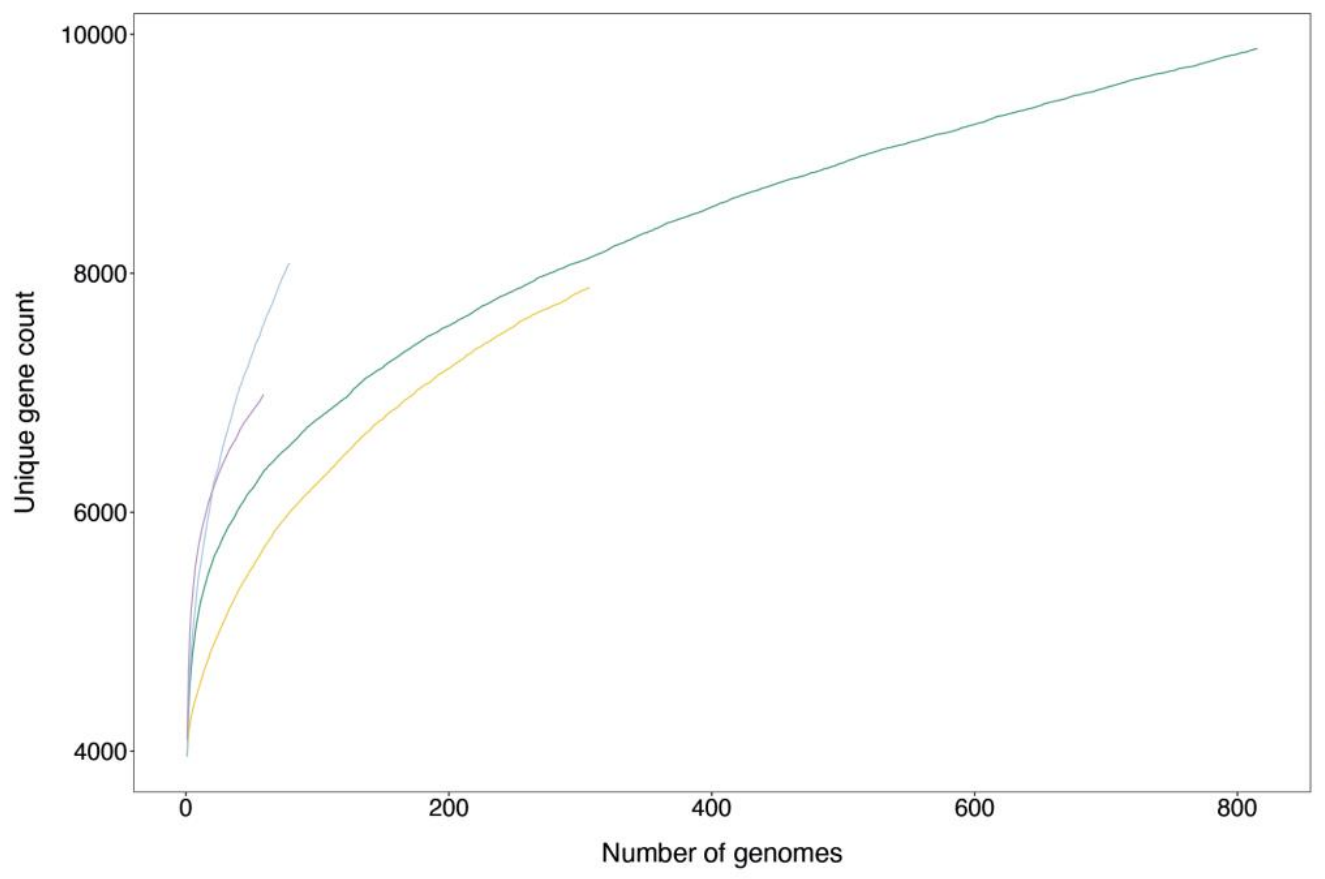

S. flexneri

S. sonnei

S. dysenteriae 
532 Fig. S1.

533 Pangenome accumulation curve of S. flexneri, S. sonnei, S. boydii and S. boydi. Each curve demonstrates

534 the number of unique protein coding genes in the pangenome as a new genome is randomly added, with the 535 number of genomes plotted along the x-axis. Random permutation of the data were subsampled 100 times, 536 in which genomes are subsampled without replacement at each iteration. The y-axis shows the minimum 537 and maximum range of unique gene count after each iteration in (A) and the mean value in (B). 

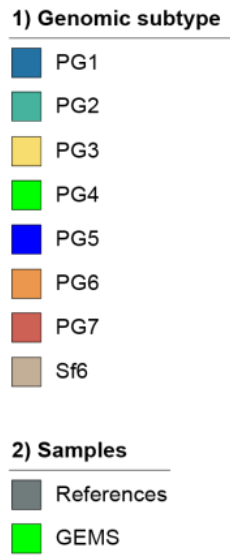

3) Geographic Location

\section{S. flexneri}

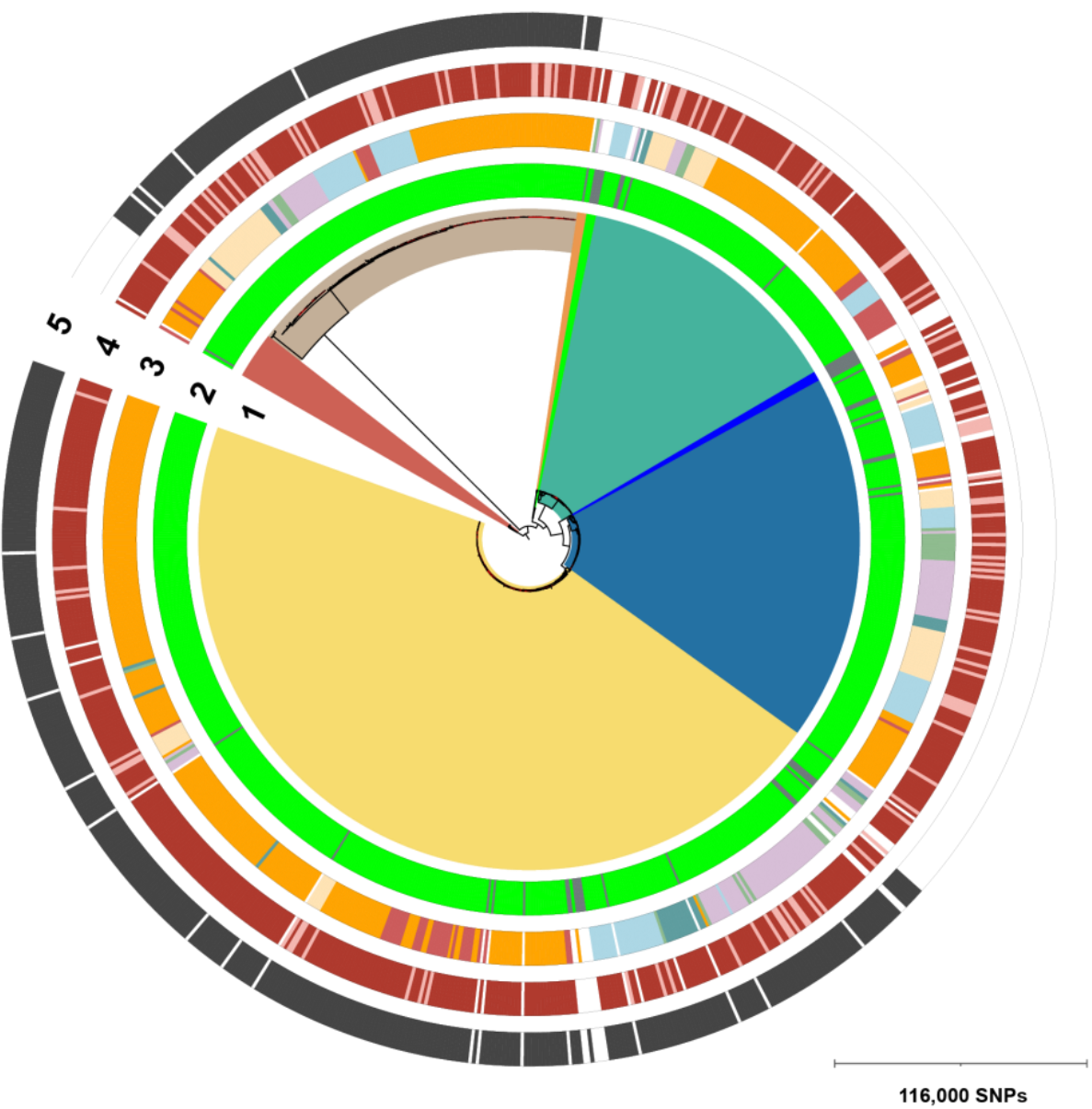

Fig. S2.

540 Phylogeny of $S$. flexneri population from GEMS. ML phylogenetic tree constructed using core genome SNPs from alignments of 817 S. flexneri genomes from GEMS and publicly available genomes. Tree was rooted using $E$. coli genome. The outer concentric rings illustrate different genotypic and epidemiological

543 data according to the numbered inlaid keys displayed next to the tree. Scale bars represents the number of 544 SNPs. 


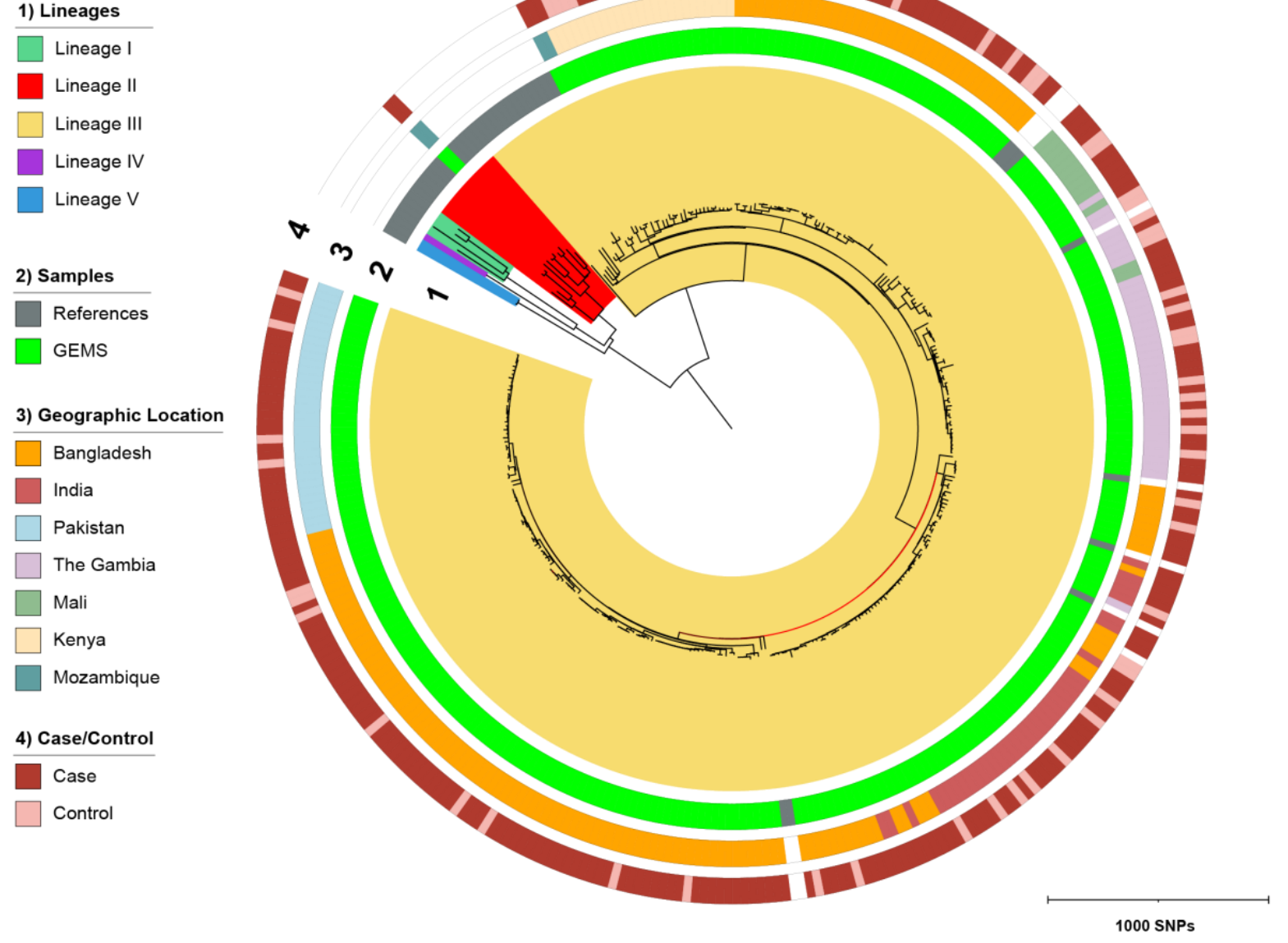

\section{S. sonnei}

Fig. S3.

547 Phylogeny of $S$. sonnei population from GEMS. Midpoint rooted ML phylogenetic tree constructed using 548 core genome SNPs from alignments of 308 S. sonnei genomes from GEMS and publicly available genomes. 
bioRxiv preprint doi: https://doi.org/10.1101/2021.06.09.447709; this version posted June 10, 2021. The copyright holder for this preprint (which was not certified by peer review) is the author/funder, who has granted bioRxiv a license to display the preprint in perpetuity. It is made available under aCC-BY 4.0 International license.

A

\section{S. boydii}
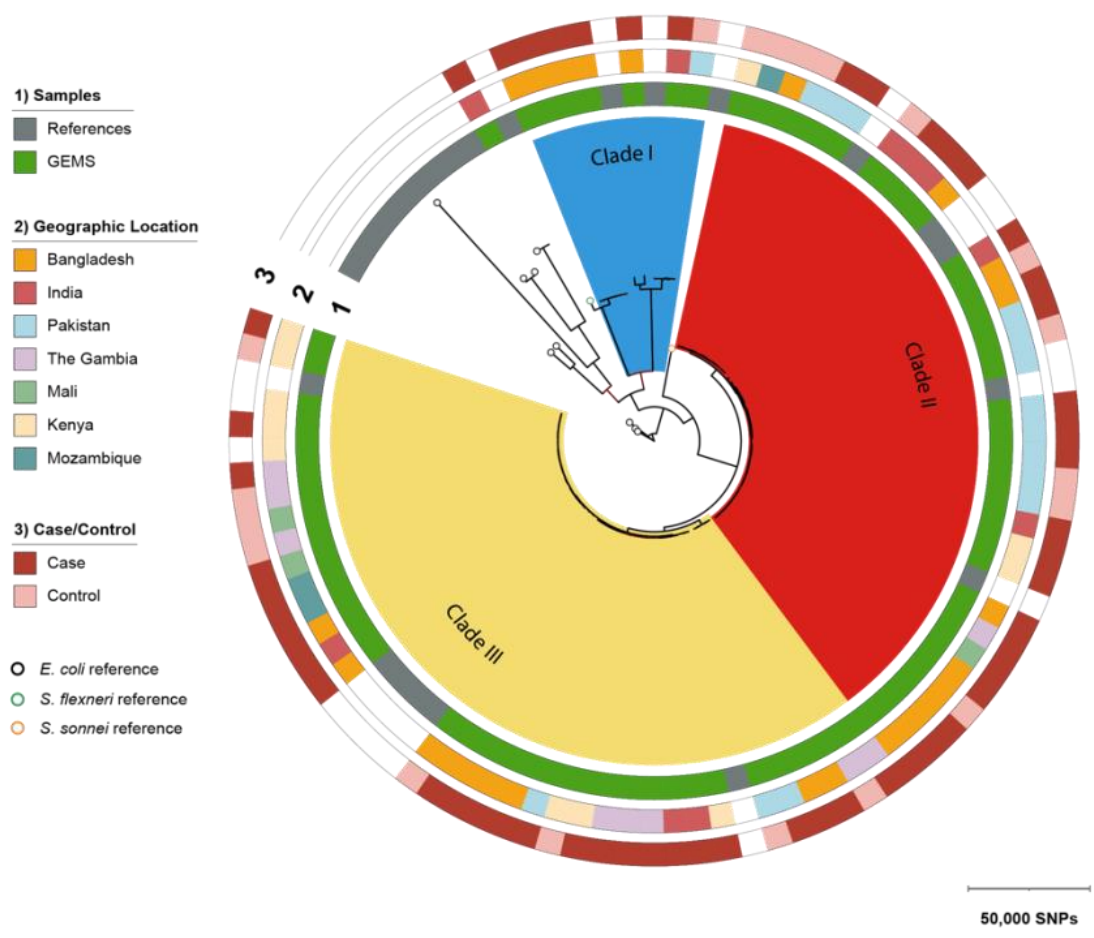

B

\section{S. dysenteriae}

\begin{tabular}{l} 
1) Samples \\
\hline$\square$ References \\
GEMS \\
2) Geographic Location \\
$\square$ Bangladesh \\
$\square$ India \\
$\square$ Pakistan \\
$\square$ The Gambia \\
$\square$ Mali \\
$\square$ Kenya \\
3) Case/Control \\
$\square$ Case \\
$\square$ Control \\
E. coli reference \\
\hline S. flexneri reference \\
\hline S. sonnei reference
\end{tabular}

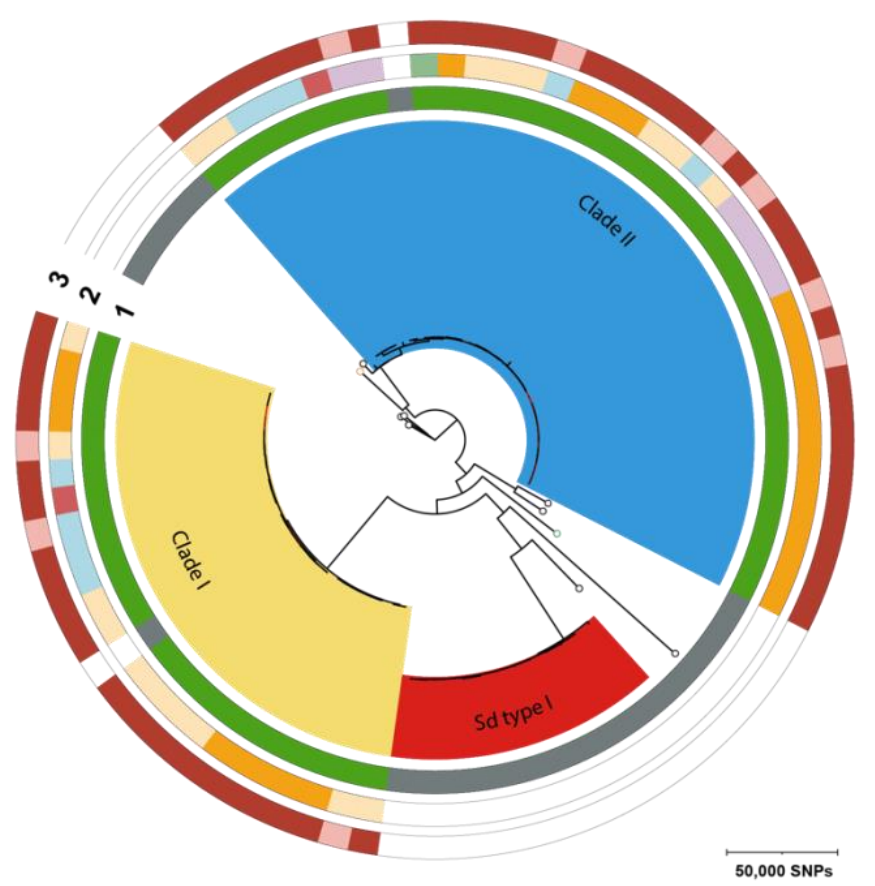




\section{$550 \quad$ Fig. S4.}

551 Phylogeny of S. boydii and S. dysenteriae population from GEMS. ML phylogenetic trees were constructed

552 based on core genome SNPs outside region of recombination from alignments of (A) 79 S. boydii and (B)

$55360 \mathrm{~S}$. dysenteriae genomes from GEMS and publicly available genomes. Both trees were rooted using $E$.

554 coli genome. Scale bar represent number of SNPs. 

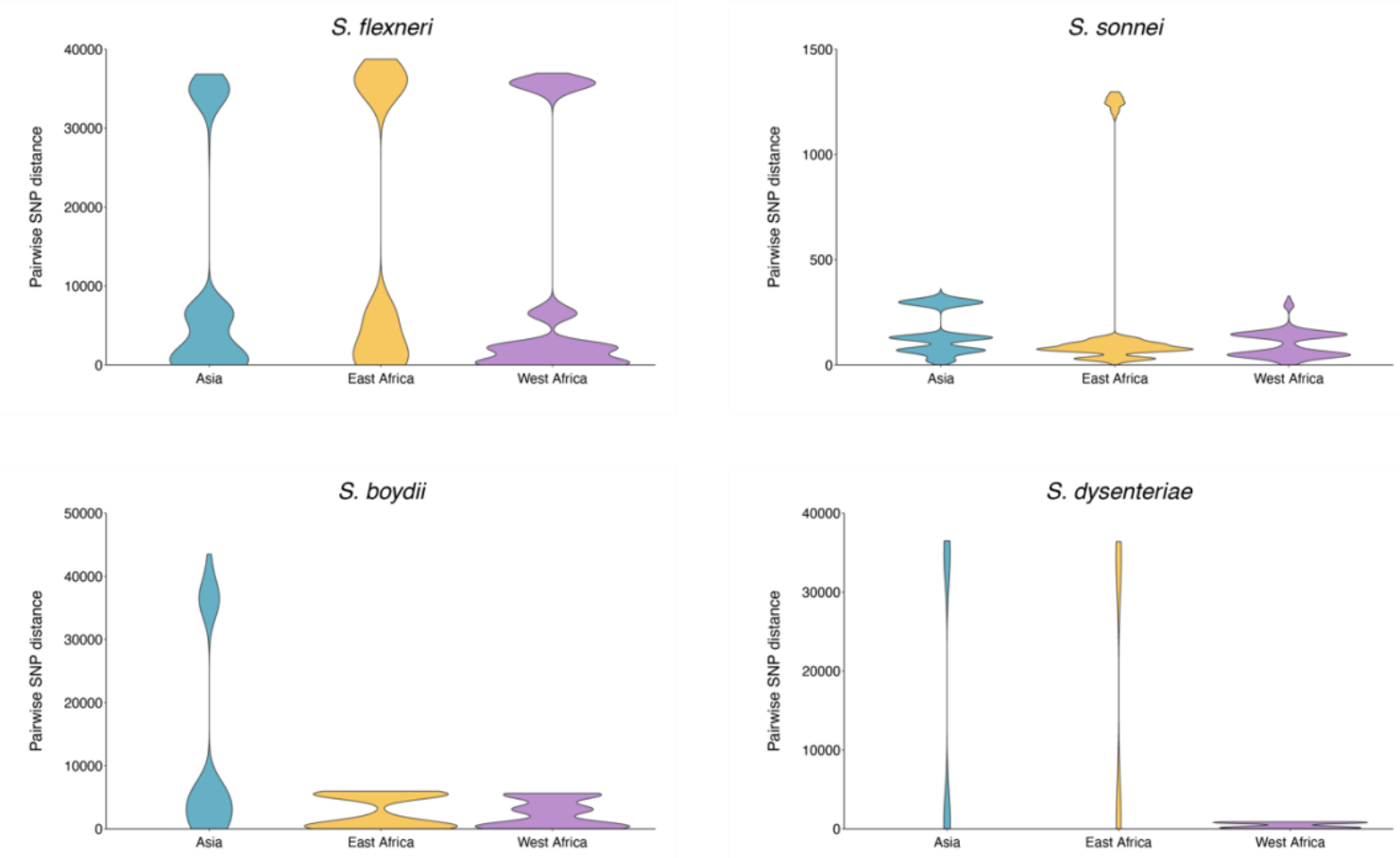

\section{$556 \quad$ Fig. S5.}

557 Regional diversity of Shigella spp. Comparison of genomic diversity, as measured by pairwise core SNP

558 distance, across GEMS study sites (Asia: Bangladesh, India and Pakistan; East Africa: Kenya and 559 Mozambique; West Africa: The Gambia and Mali) for S. flexneri, S. sonnei, S. boydii and S. dysenteriae. 
A

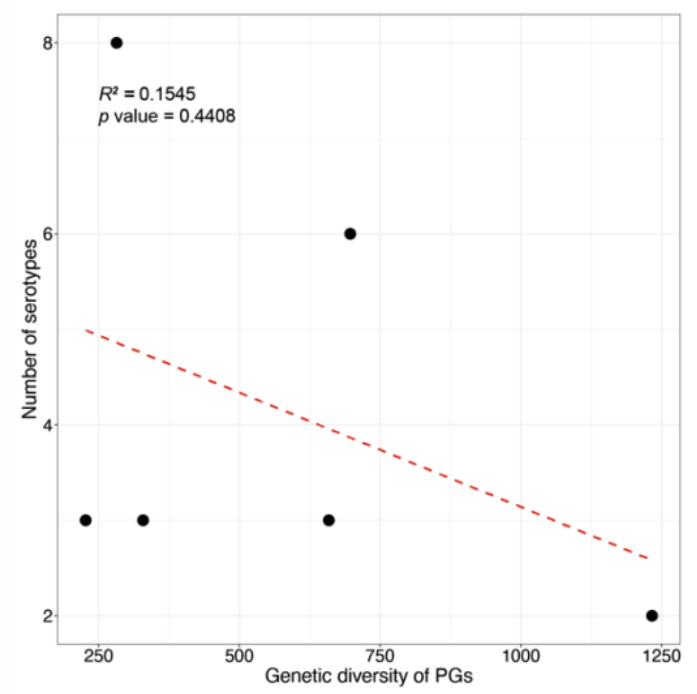

B

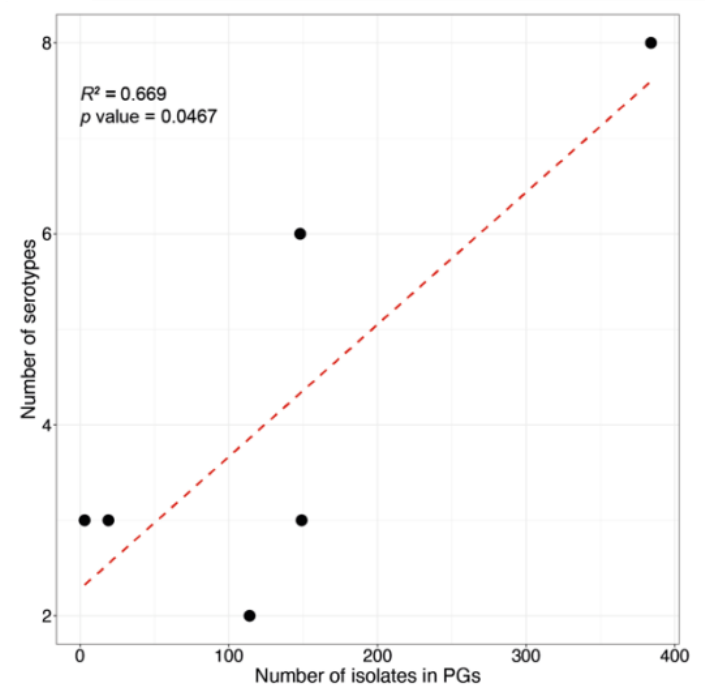

$561 \quad$ Fig. S6.

562 Association of S. flexneri serotype diversity with different properties of a genomic subtype. For each of the

563 six subtypes identified among S. flexneri (PG1-PG7 and Sf6), the number of different serotypes is displayed

564 along the $\mathrm{y}$-axis and plotted against (A) the number of isolates within the subtype and (B) the genetic

565 diversity of the subtype, as measured by pairwise core SNP distance and plotted along the x-axis. Linear

566 regression analysis was performed to assess the association between serotype diversity and the different

567 properties of subtypes. The regression coefficient of determination $\left(\mathrm{R}^{2}\right)$ and $p$-value are displayed on the

568 top left of each plot. 

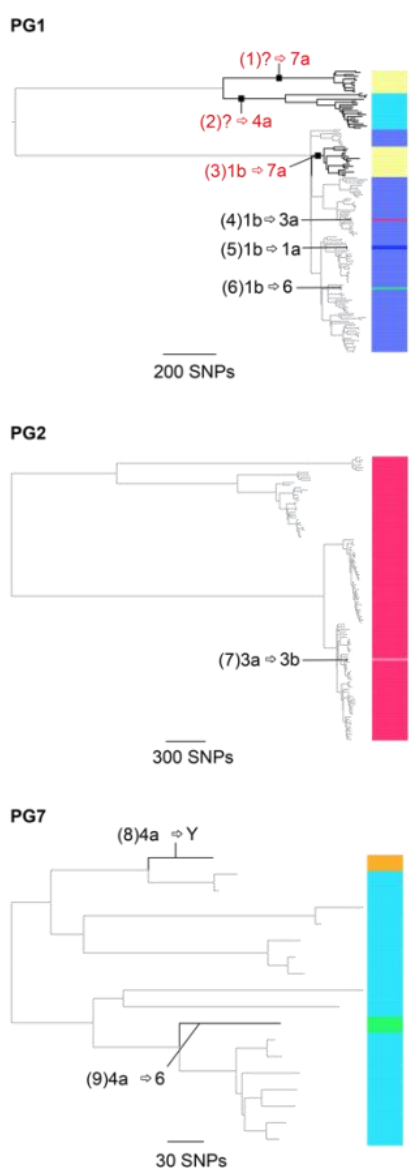
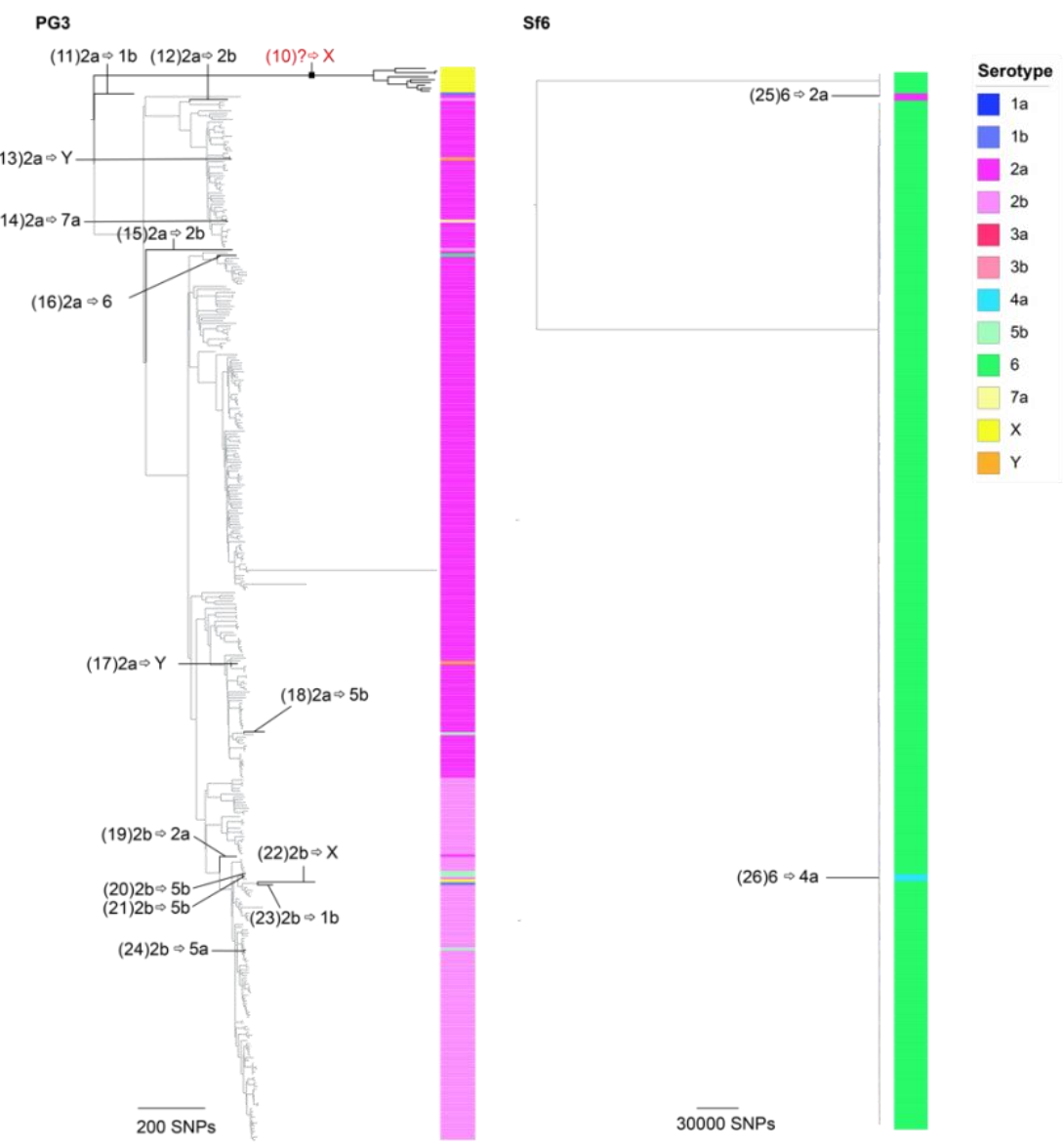

$570 \quad$ Fig. S7.

571 Serotype switching events across $S$. flexneri genomic subtypes. ML phylogenetic tree of each subtype was

572 generated based on core genome SNPs. Serotypes determined through biochemical serotyping are displayed

573 on the right-hand side of each tree, and coloured according to the inlaid key. The 26 inferred serotype

574 switching events occurring along the phylogenetic branches are labelled accordingly. Numbers inside each

575 backets represents switch IDs, with further details provided in table S3. Where the dominant serotype

576 cannot be determined, a question mark is displayed, indicating switch from unknown ancestral type.

577 Serotype switching events resulting in more than two descendant isolates are highlighted in red. 

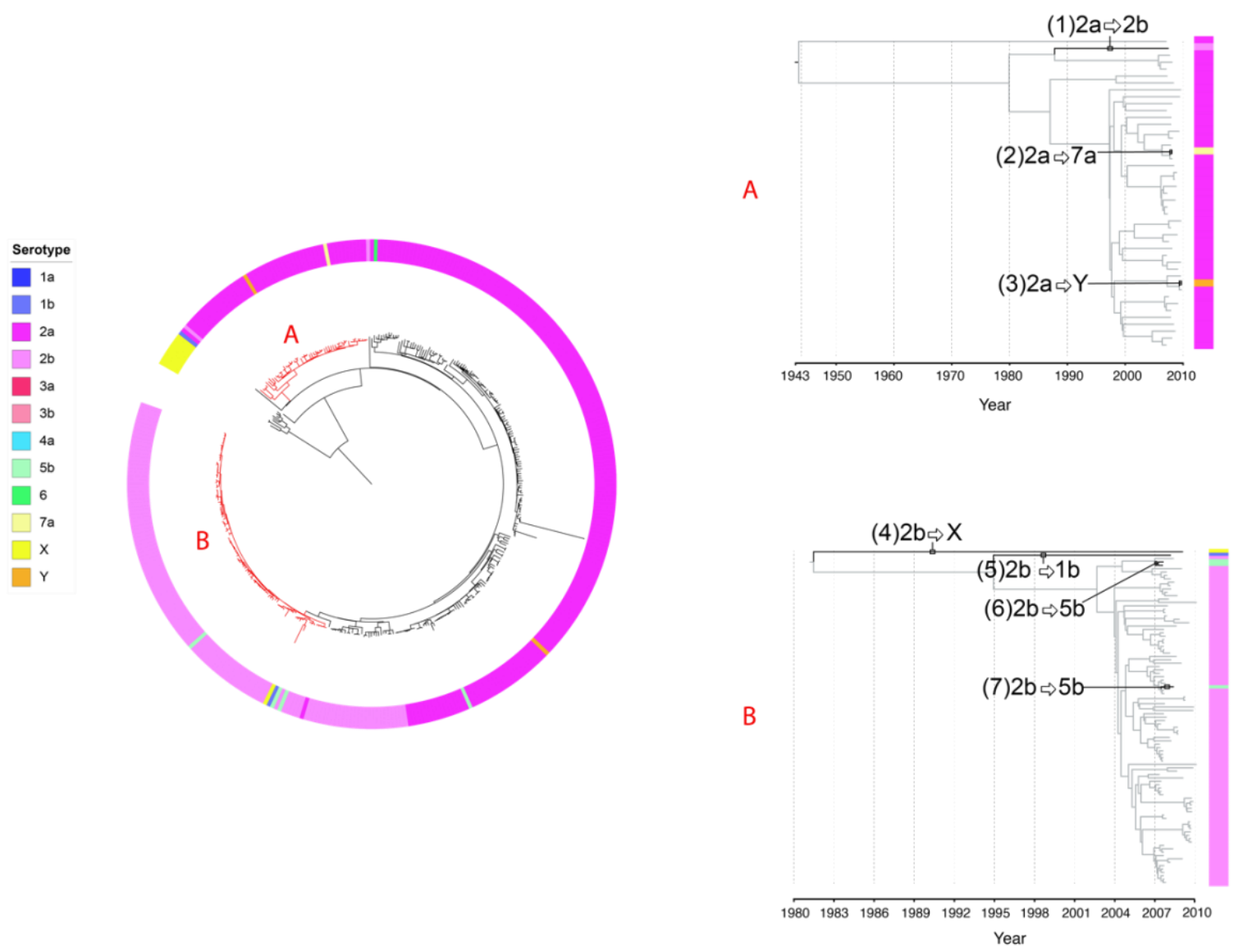

\section{$579 \quad$ Fig. S8.}

580 Estimation of time frame for serotype switching among S. flexneri PG3 isolates. ML phylogenetic tree of S. flexneri PG3 ( $n=384)$ generated using core genome SNPs is displayed on the right. Isolate serotype is displayed on the outer ring, coloured according to the inlaid key displayed next to the tree. Two subclades with branches highlighted in red were selected for BEAST analysis. Maximum clade credibility trees based on two subclades within PG3 are displayed on the left. Independent switching events occurring along the various phylogenetic branches are highlighted in black, labelled and annotated. BEAST estimated time frame of divergence along the branches of the seven isolates that have undergone serotype switching are shown in table S5. 


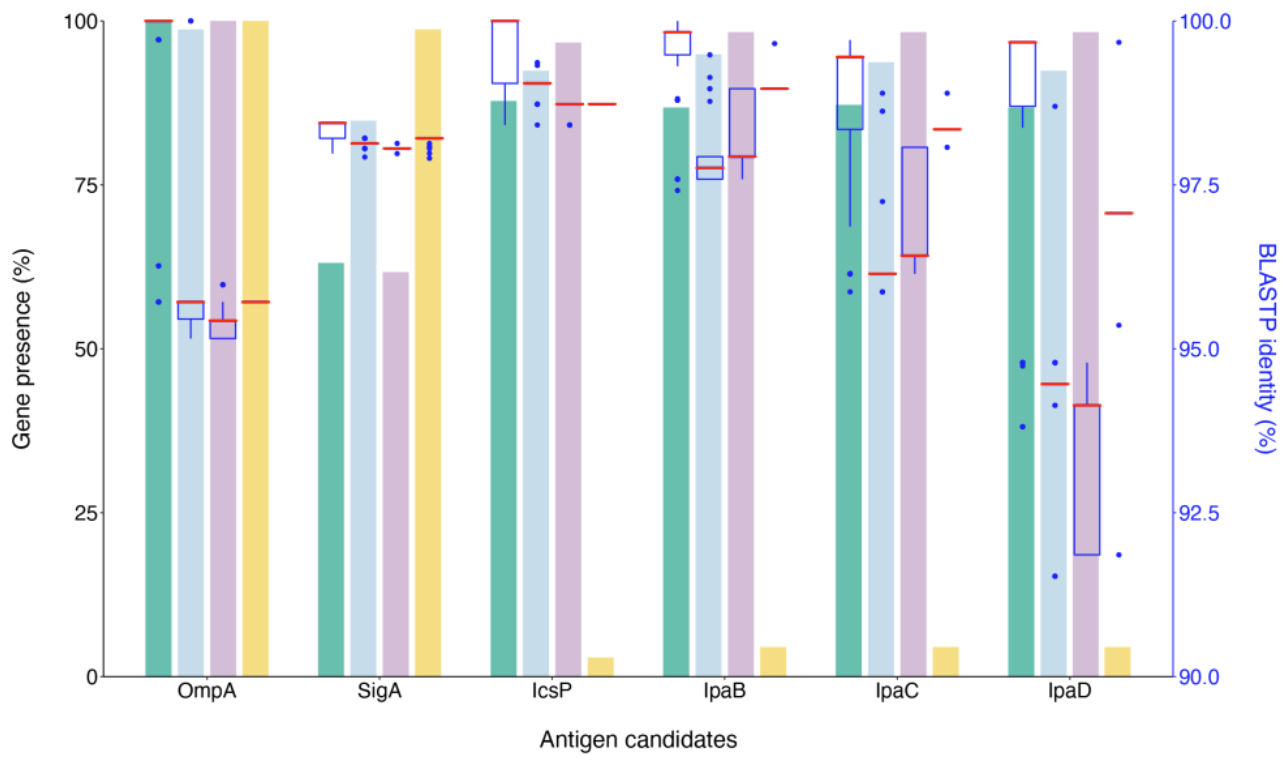

Fig. S9.

590 The distribution of vaccine antigen candidate and protein sequence identity among Shigella spp. (A)

591 Lefthand y-axis refers to the grouped bar plot displaying presence of vaccine candidate genes identified

592 among Shigella isolates from GEMS. Bars are grouped by genes and coloured according to species.

593 Righthand y-axis (blue) refers to the boxplot displaying the interquartile range, median (red) and

594 minimum/maximum pairwise percentage identity of the amino acid sequences of antigen vaccine

595 candidates among GEMS, compared against the reference sequences. Presence of genes were identified

596 using BLASTn search against draft genome assemblies and amino acid sequence percentage identity were

597 inferred using BLASTp. (B) Mapping coverage of Shigella spp. virulence plasmid. Low percentage of

598 virulence plasmid were detected among $S$. sonnei isolates, likely contributed by the fact that $S$. sonnei

599 virulence plasmid is comparatively unstable and often lost during subculturing. 

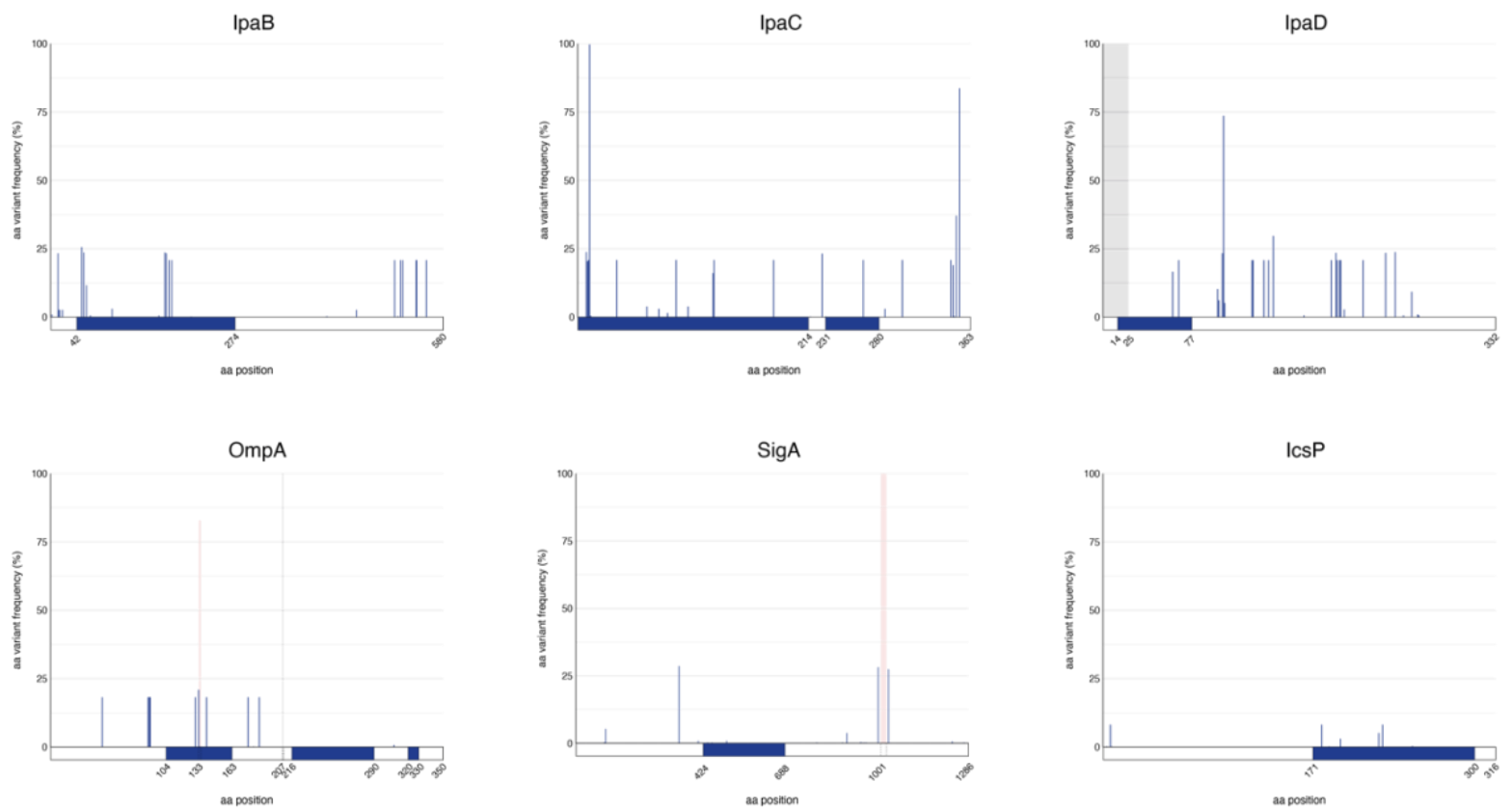

601 Fig. S10.

602 Frequency of amino acid variation among S. flexneri population for antigen vaccine candidates. Frequency of amino acid variations within $S$. flexneri genomes for the six vaccine candidate protein sequences. For each protein sequence, the proportion of genomes with the variant is shown along the y-axis with the position of the variant plotted along the x-axis. Grey bars highlight regions where there is a deletion and

606 red bars highlight insertions. Schematic of the known epitope positions (in blue) for the protein sequences are displayed below the $\mathrm{x}$-axis. 


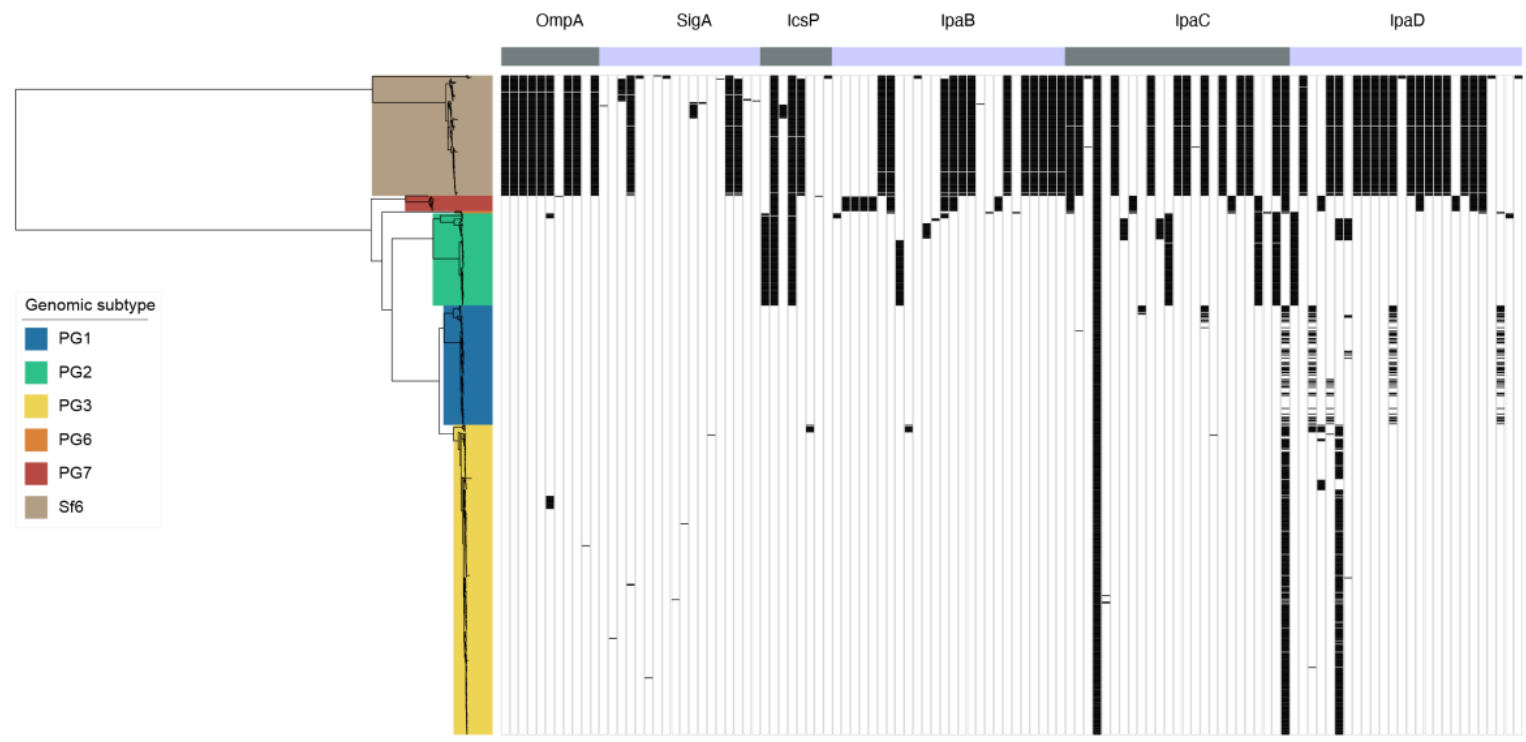

Fig. S11.

610 Vaccine antigen variation among S. flexneri subtypes. ML phylogenetic tree of $806 \mathrm{~S}$. flexneri isolates based on core genome SNPs is displayed on the left, the six subtypes identified among the population are highlighted in different colours according to the inlaid key. The alternating grey and purple colour blocks displayed above the top panel represents the six antigen vaccine candidates assessed in the current study. The matrix in the centre demonstrates presence (in black) of aa variation for each antigen vaccine. Only variable sites are displayed. 


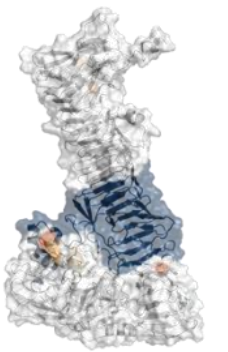

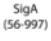

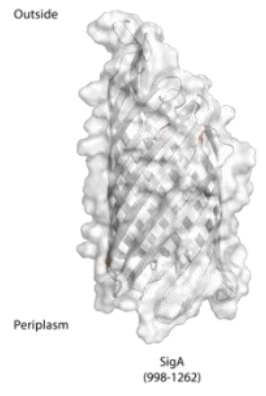

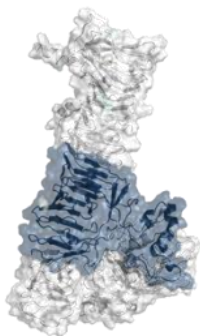

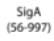

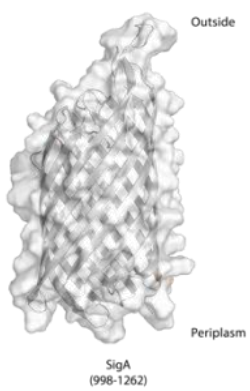

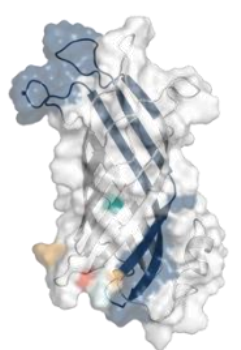

$\underset{\substack{\text { Ompes } \\ 022216}}{210}$
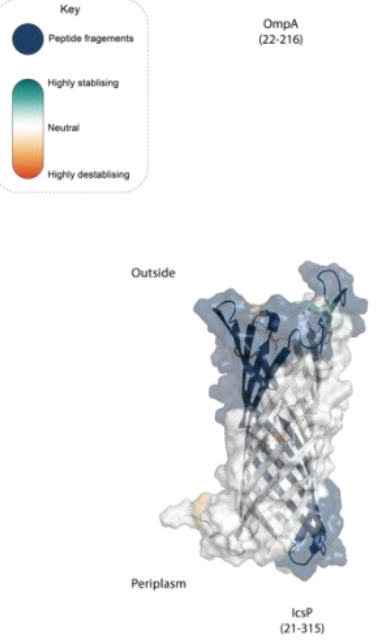

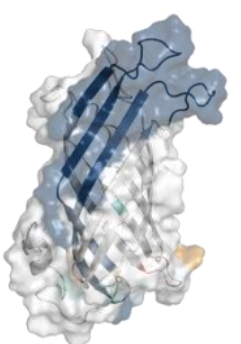

$\underset{\substack{\text { Ompen } \\(222216)}}{2}$

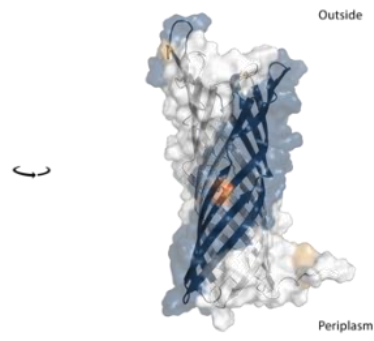

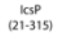

\section{Fig. S12.}

618 Visualization of mutations on modelled SigA, OmpA and IcsP protein antigens. Visualisation of mutations 619 on modelled proteins, with protein residues modelled shown in brackets. Peptide fragments for OmpA, 620 SigA and IcsP that are used for vaccine development are coloured in blue. Predicted effects of mutations 621 within the proteins are coloured using the scale shown in the key. OmpA, SigA and IcsP are orientated so that the extracellular space is located at the top of the figure, and the periplasmic space is at the bottom. 
bioRxiv preprint doi: https://doi.org/10.1101/2021.06.09.447709; this version posted June 10, 2021. The copyright holder for this preprint (which was not certified by peer review) is the author/funder, who has granted bioRxiv a license to display the preprint in perpetuity. It is made available under aCC-BY 4.0 International license.
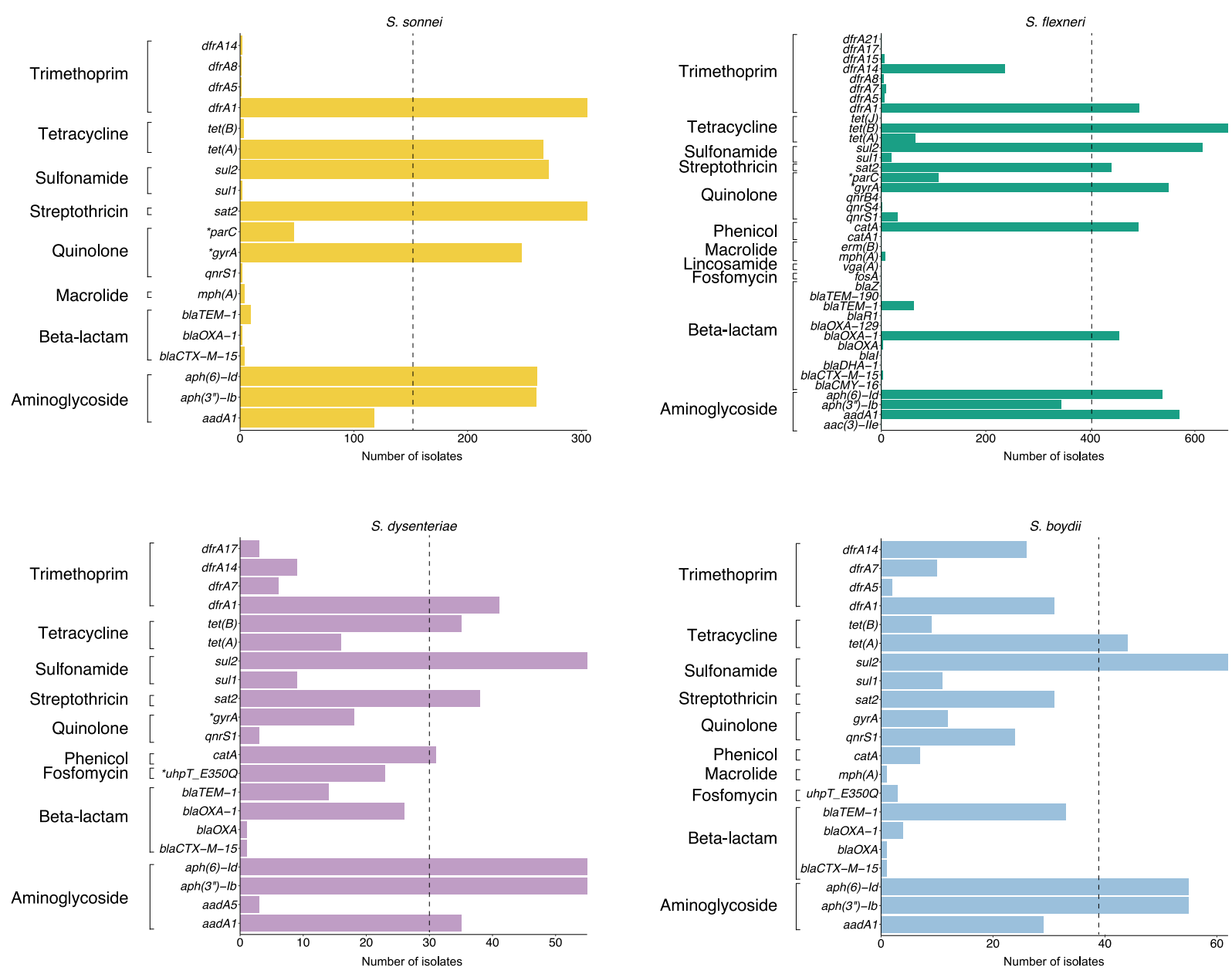

\section{Fig. S13.}

625 Prevalence of genetic determinants conferring AMR among Shigella spp. Bar plots shows the number of genetic determinants detected in S. sonnei, S. flexneri, S. dysenteriae and S. boydii isolates that confer resistance or reduced susceptibility to various antimicrobials. Genes and point mutations (indicated with an asterisk) are plotted along the $y$-axis and grouped by drug class (displayed on the left). The dashed lines highlight genetic determinants identified in half or more of the isolates for each species. 
bioRxiv preprint doi: https://doi.org/10.1101/2021.06.09.447709; this version posted June 10,2021. The copyright holder for this preprint (which was not certified by peer review) is the author/funder, who has granted bioRxiv a license to display the preprint in perpetuity. It is made available under aCC-BY 4.0 International license.

A
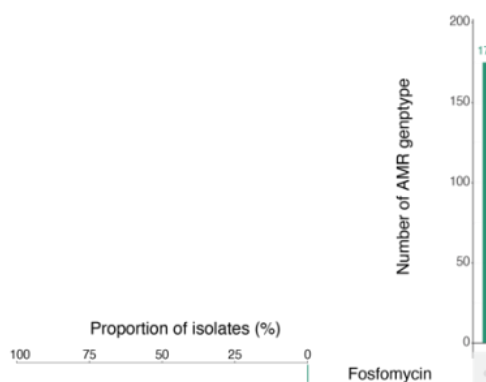

S. flexneri

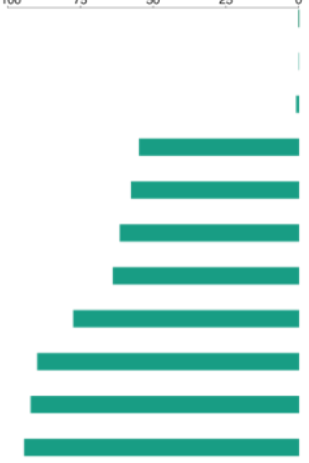

Lincosamide

150

Streptothricin

Quinolone

Phenicol

Beta-lactam

Sulfonamide

Tetracycline

Trimethoprim

Aminoglycoside

B

Proportion of isolates (\%)

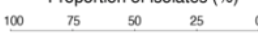
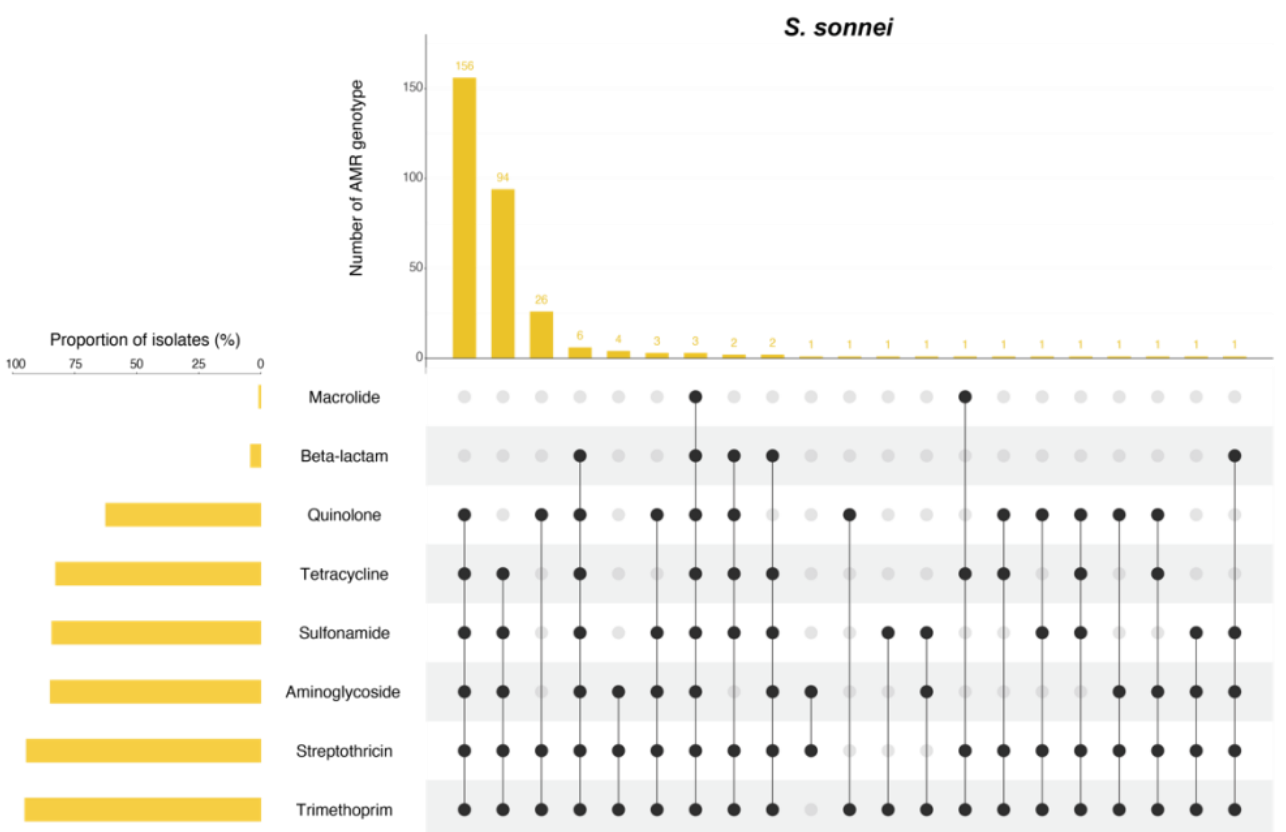
bioRxiv preprint doi: https://doi.org/10.1101/2021.06.09.447709; this version posted June 10, 2021. The copyright holder for this preprint (which was not certified by peer review) is the author/funder, who has granted bioRxiv a license to display the preprint in perpetuity. It is made available under aCC-BY 4.0 International license.

C \begin{tabular}{l} 
Proportion of isolates (\%) \\
$80 \quad 60 \quad 40 \quad 20$ \\
\hline
\end{tabular}

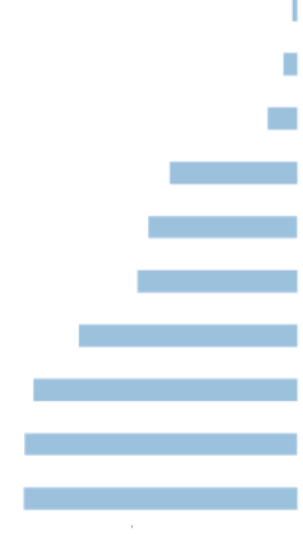

D

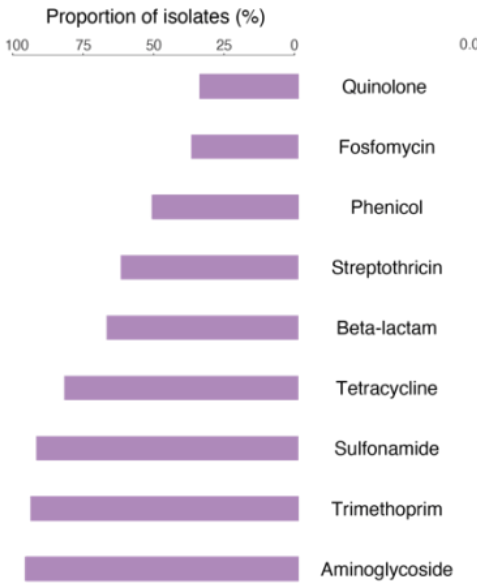

Macrolide

Fosfomycin

Phenicol

Streptothricin

Quinolone

Beta-lactam

Tetracycline

Sulfonamide

Aminoglycoside

Trimethoprim
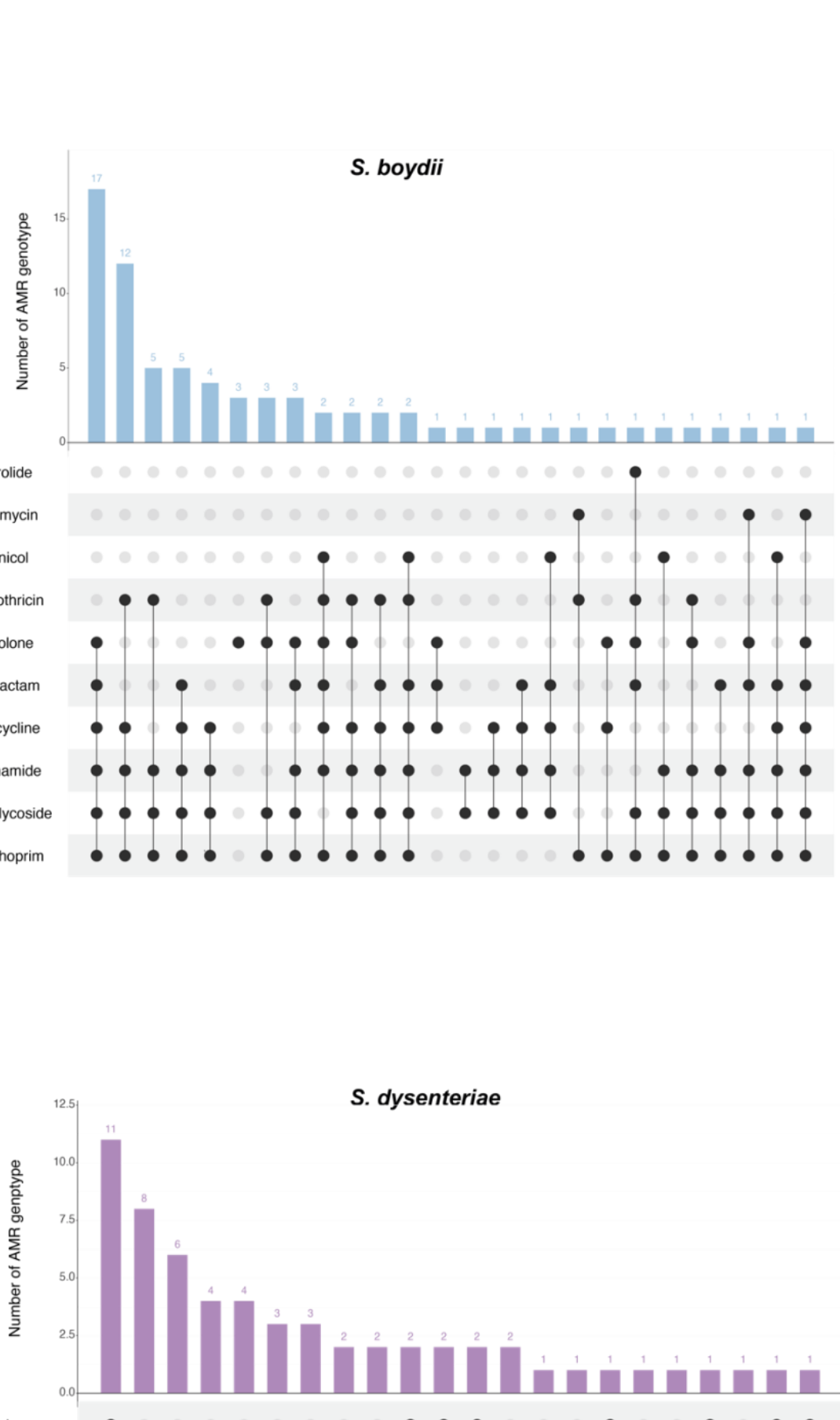

S. dysenteriae

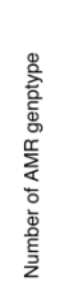
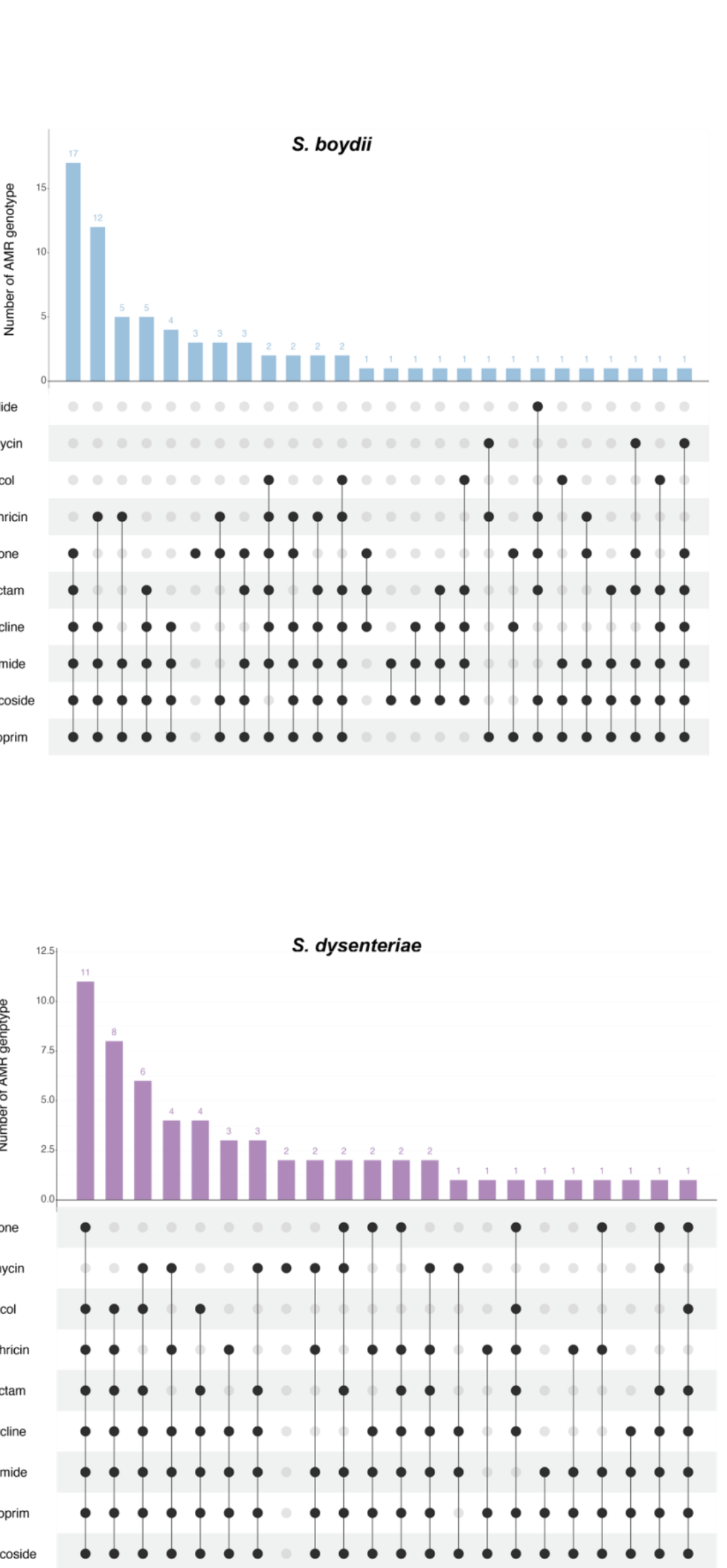


\section{Fig. S14.}

634 Diversity of AMR genotype resistance profiles. UpSet plots illustrate the AMR genotype resistance profiles

635 for (A) S. flexneri, (B) S. sonnei, (C) S. boydii and (D) S. dysenteriae. Genotypic AMR profiles are shown 636 in the combination matrix in the center panel. Each column represents a unique genotypic profile, where

637 each black dot represents presence of a genetic determinant conferring resistance or reduced susceptibility

638 to a drug class (displayed on the left). The vertical the bar plot above the matrix displays the number of 639 isolates with a particular profile, with the exact number of isolates displayed above each bar. The horizontal

640 bar plot on the left of the matrix illustrates the proportion of isolates containing AMR genetic determinants

641 associated with a drug class. 
A

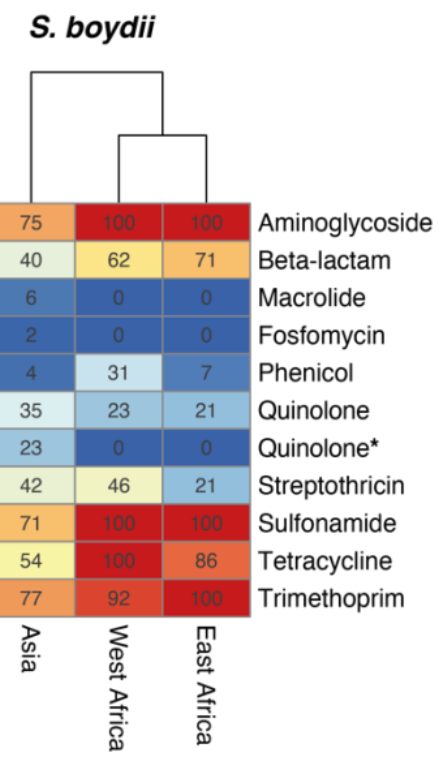

\section{S. dysenteriae}

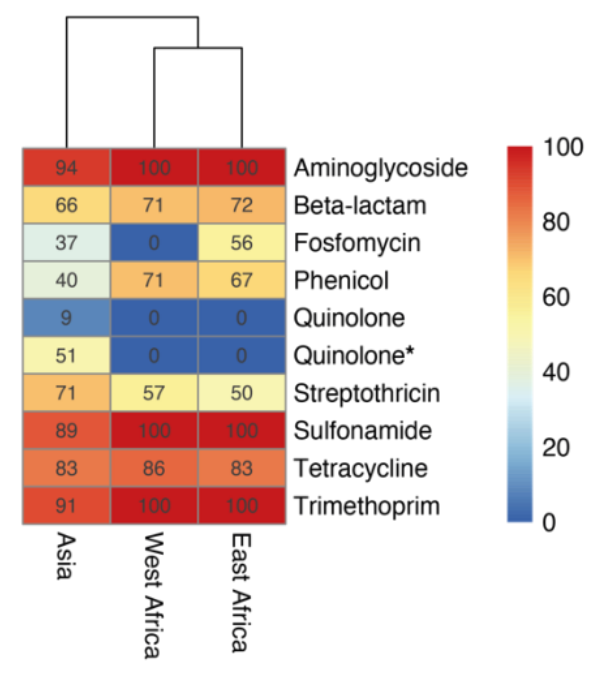

B
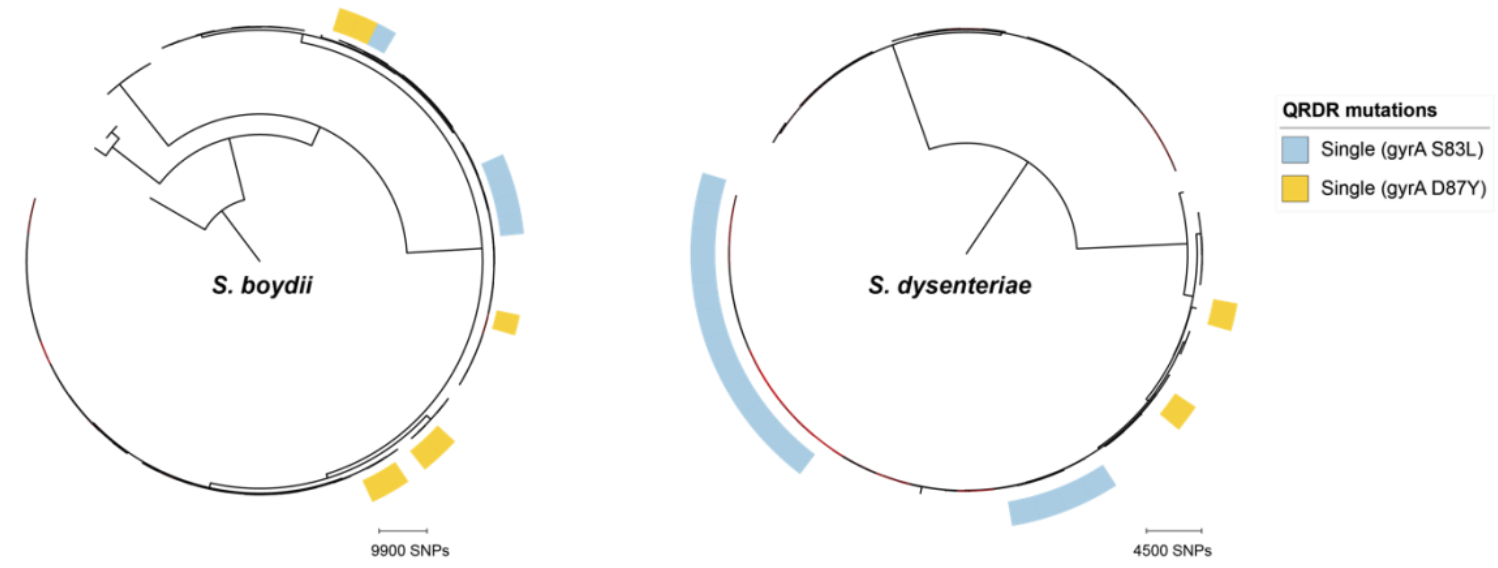

Fig. S15.

644 Detection of known AMR genetic determinants conferring resistance (reduced susceptibility marked with 645 asterisk) to various drug class, grouped by region (A) and convergent evolution of ciprofloxacin resistance 646 (B) for S. boydii and S. dysenteriae. 

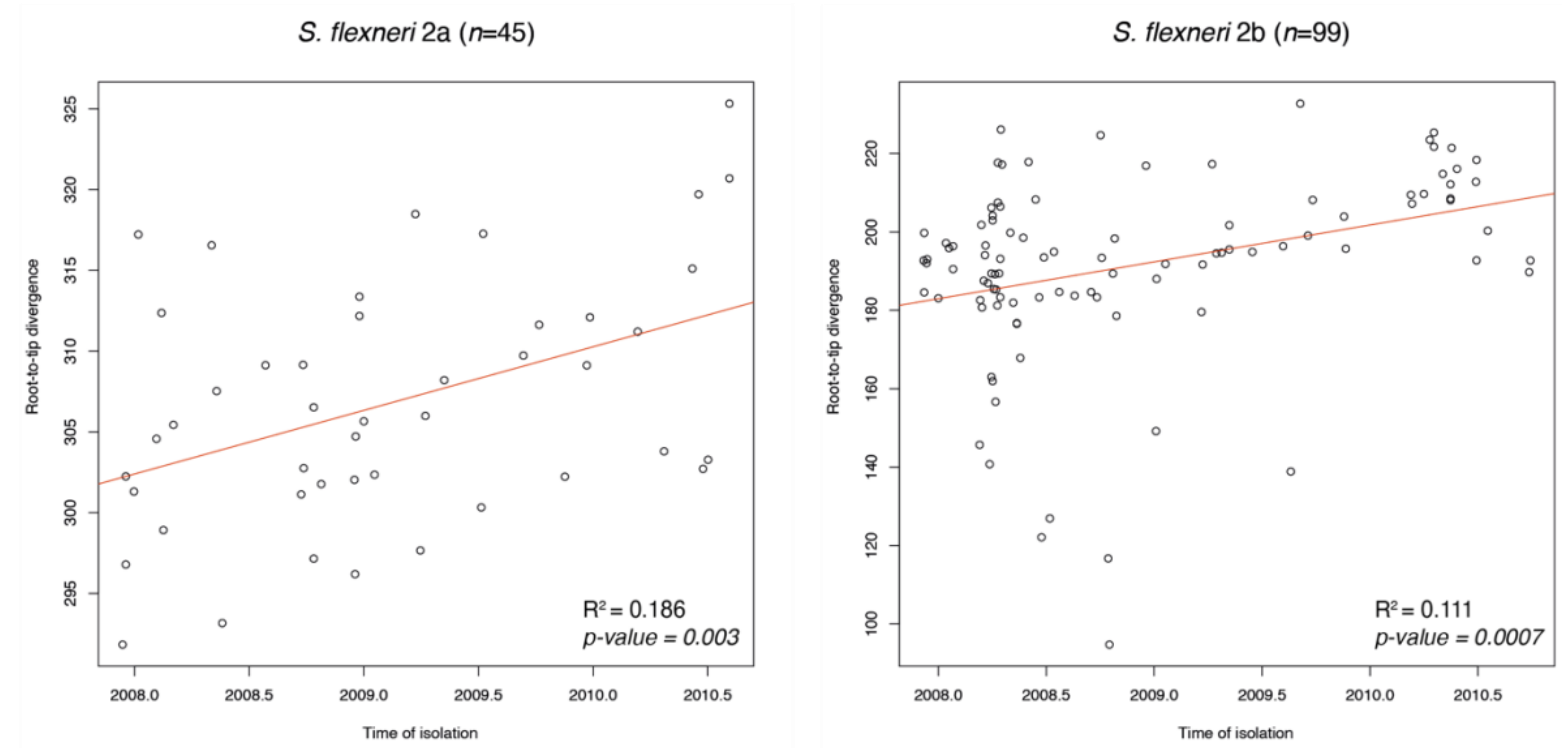

647

648 Fig. $\mathbf{S 1 6 .}$

649 Temporal phylogenetic signal for S. flexneri. Correlation between isolate sampling time in months (x-axis) 650 and phylogenetic root-to-tip divergence (y-axis), as estimated by TempEst based on ML phylogeny of each 651 subclade. The two datasets correspond to S. flexneri 2a isolates belonging to node A (left) and S. flexneri 652 2b isolates belonging to node B (right) from PG3 in fig. S8. The linear regression line is coloured in red, 653 with the coefficient of determination $\left(\mathrm{R}^{2}\right)$ and $p$-value displayed for each plot. 
654 Table S1.

655 Details of Shigella isolates used in this study. Includes accession numbers of the sequencing reads used 656 in the study, Shigella serotype, assembly statistics, year and country of isolation, condition of the child 657 (case/control) from which the isolate was derived from as defined by GEMS, genomic subtype, AMR genes 658 and QRDR mutations.

659

660 See separate Excel file 
661 Table S2.

662 Details of publicly available E.coli/Shigella genomes used in this study.

\begin{tabular}{|c|c|c|c|}
\hline Accession & strain & $\begin{array}{l}\text { Species / } \\
\text { serotype }\end{array}$ & Phylogroup/Lineage/subtype \\
\hline ERR028677 & 5417_1\#4 & S. sonnei & Central Asia III \\
\hline ERR028679 & 5417_1\#6 & S. sonnei & Central Asia III \\
\hline ERR024610 & $5008 \_7 \# 5$ & S. sonnei & Central Asia III \\
\hline ERR028705 & 5417_3\#8 & S. sonnei & Central Asia III \\
\hline ERR024611 & $5008 \_7 \# 6$ & S. sonnei & Central Asia III \\
\hline ERR200544 & 8403_8\#89 & S. sonnei & $\mathrm{V}$ \\
\hline ERR200550 & $8403 \_8 \# 95$ & S. sonnei & $\mathrm{V}$ \\
\hline ERR025737 & $5236 \_6 \# 2$ & S. sonnei & IV \\
\hline ERR025768 & $5236 \_8 \# 9$ & S. sonnei & Global III \\
\hline ERR316396 & 9803_4\#91 & S. sonnei & Global III \\
\hline ERR200471 & $8403 \_8 \# 16$ & S. sonnei & Global III \\
\hline ERR025765 & $5236 \_8 \# 6$ & S. sonnei & I \\
\hline ERR025722 & $5236 \_5 \# 10$ & S. sonnei & $\mathrm{I}$ \\
\hline ERR024606 & 5008_7\#11 & S. sonnei & I \\
\hline ERR025754 & $5236 \_7 \# 7$ & S. sonnei & I \\
\hline ERR025735 & $5236 \_6 \# 10$ & S. sonnei & II \\
\hline ERR025726 & 5236_5\#3 & S. sonnei & II \\
\hline ERR028675 & 5417_1\#2 & S. sonnei & II \\
\hline ERR028673 & 5417_1\#11 & S. sonnei & II \\
\hline ERR025751 & 5236_7\#4 & S. sonnei & II \\
\hline ERR025762 & $5236 \_8 \# 3$ & S. sonnei & II \\
\hline ERR025689 & 5236_1\#5 & S. sonnei & II \\
\hline ERR025692 & $5236 \_1 \# 8$ & S. sonnei & II \\
\hline ERR025724 & $5236 \_5 \# 12$ & S. sonnei & II \\
\hline ERR028700 & $5417 \_3 \# 3$ & S. sonnei & II \\
\hline ERR028688 & 5417_2\#2 & S. sonnei & III \\
\hline ERR025747 & $5236 \_7 \# 10$ & S. sonnei & III \\
\hline ERR025749 & $5236 \_7 \# 2$ & S. sonnei & III \\
\hline ERR025702 & 5236_2\#5 & S. sonnei & III \\
\hline ERR025700 & 5236_2\#3 & S. sonnei & III \\
\hline ERR025701 & $5236 \_2 \# 4$ & S. sonnei & III \\
\hline ERR025748 & $5236 \_7 \# 11$ & S. sonnei & III \\
\hline ERR028695 & 5417_2\#9 & S. sonnei & III \\
\hline ERR025698 & $5236 \_2 \# 12$ & S. sonnei & III \\
\hline ERR316322 & 9803_4\#17 & S. sonnei & Latin America IIIa \\
\hline ERR212328 & 8489_1\#60 & S. sonnei & Latin America IIIa \\
\hline ERR316241 & 9789_6\#32 & S. sonnei & Latin America IIIa \\
\hline ERR025767 & 5236_8\#8 & S. sonnei & OJCA \\
\hline ERR190834 & $8290 \_4 \# 28$ & S. sonnei & OJCA \\
\hline ERR319257 & 9870_7\#10 & S. sonnei & OJCA \\
\hline
\end{tabular}




\begin{tabular}{|c|c|c|c|}
\hline NC_007384 & $\mathrm{Ss} 046$ & S. sonnei & III \\
\hline LVIU01000110.1 & ASM164910v1 & S. flexneri 4s & \\
\hline NZ_CM001474.1 & M90T & S. flexneri $5 \mathrm{a}$ & \\
\hline NC_004741.1 & $2457 \mathrm{~T}$ & S. flexneri $2 \mathrm{a}$ & \\
\hline ERR042803 & ERR042803 & S. flexneri $2 \mathrm{a}$ & Phylogroup 3 \\
\hline ERR042850 & ERR042850 & S. flexneri $2 \mathrm{a}$ & Phylogroup 3 \\
\hline ERR048281 & ERR048281 & S. flexneri & Phylogroup 2 \\
\hline ERR048288 & ERR048288 & S. flexneri & Phylogroup 6 \\
\hline ERR048302 & ERR048302 & S. flexneri $2 \mathrm{a}$ & Phylogroup 3 \\
\hline ERR048305 & ERR048305 & S. flexneri & Phylogroup 1 \\
\hline ERR048317 & ERR048317 & S. flexneri & Phylogroup 7 \\
\hline ERR048339 & ERR048339 & S. flexneri $2 \mathrm{a}$ & Phylogroup 3 \\
\hline ERR126987 & ERR126987 & S. flexneri $2 \mathrm{a}$ & Phylogroup 3 \\
\hline ERR126993 & ERR126993 & S. flexneri & Phylogroup 2 \\
\hline ERR127032 & ERR127032 & S. flexneri 1a & PHE type strain \\
\hline ERR127033 & ERR127033 & S. flexneri $1 \mathrm{~b}$ & PHE type strain \\
\hline ERR127034 & ERR127034 & S. flexneri 1c & PHE type strain \\
\hline ERR127035 & ERR127035 & S. flexneri $2 \mathrm{a}$ & PHE type strain \\
\hline ERR127036 & ERR127036 & S. flexneri $2 \mathrm{~b}$ & PHE type strain \\
\hline ERR127037 & ERR127037 & S. flexneri 3a & PHE type strain \\
\hline ERR127038 & ERR127038 & S. flexneri $3 \mathrm{~b}$ & PHE type strain \\
\hline ERR127039 & ERR127039 & S. flexneri 3c & PHE type strain \\
\hline ERR127040 & ERR127040 & S. flexneri $4 \mathrm{a}$ & PHE type strain \\
\hline ERR127041 & ERR127041 & S. flexneri 4b & PHE type strain \\
\hline ERR127043 & ERR127043 & S. flexneri $5 \mathrm{a}$ & PHE type strain \\
\hline ERR127044 & ERR127044 & S. flexneri $5 \mathrm{~b}$ & PHE type strain \\
\hline ERR127046 & ERR127046 & S. flexneri & Phylogroup 4 \\
\hline ERR127047 & ERR127047 & S. flexneri Y & PHE type strain \\
\hline ERR127048 & ERR127048 & $\begin{array}{l}\text { S. flexneri } \\
\text { E1037 }\end{array}$ & PHE type strain \\
\hline ERR1363976 & ERR1363976 & S. flexneri $2 \mathrm{a}$ & Phylogroup 3 Central Asia \\
\hline ERR1364007 & ERR1364007 & S. flexneri $2 \mathrm{a}$ & Phylogroup 3 Central Asia \\
\hline ERR1364014 & ERR1364014 & S. flexneri $2 \mathrm{a}$ & $\begin{array}{l}\text { Phylogroup } 3 \text { Minor MSM } \\
\text { clade }\end{array}$ \\
\hline ERR1364050 & ERR1364050 & S. flexneri $2 \mathrm{a}$ & $\begin{array}{l}\text { Phylogroup } 3 \text { Minor MSM } \\
\text { clade }\end{array}$ \\
\hline ERR1364087 & ERR1364087 & S. flexneri $2 \mathrm{a}$ & Phylogroup 3 Central Asia \\
\hline ERR1364097 & ERR1364097 & S. flexneri $2 \mathrm{a}$ & Phylogroup 1 Central Asia \\
\hline ERR1364106 & ERR1364106 & S. flexneri $2 \mathrm{a}$ & $\begin{array}{l}\text { Phylogroup } 3 \text { Major MSM } \\
\text { clade }\end{array}$ \\
\hline ERR1364137 & ERR1364137 & S. flexneri $2 \mathrm{a}$ & $\begin{array}{l}\text { Phylogroup } 3 \text { Major MSM } \\
\text { clade }\end{array}$ \\
\hline ERR200376 & ERR200376 & S. flexneri $2 \mathrm{a}$ & Phylogroup 3 \\
\hline ERR217085 & ERR217085 & S. flexneri & Phylogroup 1 \\
\hline
\end{tabular}




\begin{tabular}{|c|c|c|c|}
\hline ERR449043 & ERR449043 & S. flexneri 3a & MSM-outbreak associated \\
\hline ERR449077 & ERR449077 & S. flexneri 3a & MSM-outbreak associated \\
\hline ERR559526 & ERR559526 & S. flexneri $2 \mathrm{a}$ & NCTC1 \\
\hline ERR832464 & ERR832464 & S. flexneri & Phylogroup 5 \\
\hline ERR832481 & ERR832481 & S. flexneri & Phylogroup 3 \\
\hline SRR7886341 & SRR7886341 & S. flexneri & MSM associated \\
\hline NC_017328.1 & ASM2224v1 & S. flexneri & \\
\hline NC_004337 & $\begin{array}{l}\text { S. flexneri } 2 \text { a str. } \\
301\end{array}$ & S. flexneri $2 a$ & Phylogroup 3 \\
\hline NC_007606 & $\begin{array}{l}\text { S. dysenteriae } \\
\text { Sd197 }\end{array}$ & S. dysenteriae & \\
\hline ERR1013692 & ERR1013692 & $\begin{array}{l}\text { S. dysenteriae } \\
\text { type } 1\end{array}$ & IV \\
\hline ERR1013770 & ERR1013770 & $\begin{array}{l}\text { S. dysenteriae } \\
\text { type } 1\end{array}$ & IIIa \\
\hline ERR1014006 & ERR1014006 & $\begin{array}{l}\text { S. dysenteriae } \\
\text { type } 1\end{array}$ & IIId \\
\hline ERR1014139 & ERR1014139 & $\begin{array}{l}\text { S. dysenteriae } \\
\text { type } 1\end{array}$ & IIIc \\
\hline ERR1014187 & ERR1014187 & $\begin{array}{l}\text { S. dysenteriae } \\
\text { type } 1\end{array}$ & IV \\
\hline ERR1014220 & ERR1014220 & $\begin{array}{l}\text { S. dysenteriae } \\
\text { type } 1\end{array}$ & II \\
\hline ERR1014530 & ERR1014530 & $\begin{array}{l}\text { S. dysenteriae } \\
\text { type } 1\end{array}$ & IIIc \\
\hline ERR1014532 & ERR1014532 & $\begin{array}{l}\text { S. dysenteriae } \\
\text { type } 1\end{array}$ & IIId \\
\hline ERR1014536 & ERR1014536 & $\begin{array}{l}\text { S. dysenteriae } \\
\text { type } 1\end{array}$ & I \\
\hline ERR1014541 & ERR1014541 & $\begin{array}{l}\text { S. dysenteriae } \\
\text { type } 1\end{array}$ & II \\
\hline ERR1014551 & ERR1014551 & $\begin{array}{l}\text { S. dysenteriae } \\
\text { type } 1\end{array}$ & IIIb \\
\hline ERR279284 & ERR279284 & $\begin{array}{l}\text { S. dysenteriae } \\
\text { type } 1\end{array}$ & II \\
\hline GCA_000268105 & SD_225-75 & $\begin{array}{l}\text { S. dysenteriae } \\
\text { type } 2\end{array}$ & S1 \\
\hline GCF_000815495 & SD_S6205 & $\begin{array}{l}\text { S. dysenteriae } \\
\text { type } 2\end{array}$ & S3 \\
\hline ERR200454 & SB_K-11124 & $\begin{array}{l}\text { S. boydii (ST } \\
1767)\end{array}$ & \\
\hline NZ_AMKG01000009 & $248-1 B$ & S. boydii & 3 \\
\hline NC_010658 & $3083-94$ & S. boydii & 2 \\
\hline AMJZ00000000 & SB_08_0009 & S. boydii & 3 \\
\hline AMKA00000000 & SB_08_0280 & S. boydii & 2 \\
\hline
\end{tabular}




\begin{tabular}{|c|c|c|c|}
\hline AMKB00000000 & SB_08_2671 & S. boydii & 3 \\
\hline AMKC00000000 & SB_08_2675 & S. boydii & 2 \\
\hline AMKD00000000 & SB_08_6341 & S. boydii & 2 \\
\hline AMKE00000000 & SB_09_0344 & S. boydii & 2 \\
\hline AFGC00000000 & SB_3594-74 & S. boydii & 3 \\
\hline AKNB00000000 & SB_4444-74 & S. boydii & 3 \\
\hline AFGE00000000 & SB_5216-82 & S. boydii & 1 \\
\hline AKNA00000000 & SB_965-58 & S. boydii & 1 \\
\hline AMJX00000000 & SB_S7334 & S. boydii & 3 \\
\hline NC_010658 & $3083-94$ & S. boydii & \\
\hline AAJT00000000 & B7A & E. coli & \\
\hline AM946981 & BL21(DE3) & E. coli $\mathrm{O} 7$ & \\
\hline NC_009801 & E24377A & $\begin{array}{l}\text { E. coli } \\
\text { O139:H28 }\end{array}$ & \\
\hline SRR2169557 & IAI1-117 & E. coli $\mathrm{O} 8$ & \\
\hline SRR306102 & K12-W3110 & E. coli $\mathrm{O} 16$ & \\
\hline NC_011751 & UMN026 & $\begin{array}{l}\text { E. coli } \\
\text { O17:K52:H18 }\end{array}$ & \\
\hline NC_013941 & CB9615 & E. coli $\mathrm{O} 55: \mathrm{H} 7$ & \\
\hline
\end{tabular}


664 Table S3.

665 Association of $S$. flexneri genomic subtype / serotype with case status.

\begin{tabular}{|l|l|l|l|l|}
\hline $\begin{array}{l}\text { Genomic subtype / } \\
\text { serotype }\end{array}$ & OR & 95\% CI & z statistic & p-value \\
\hline Sf6 & 0.5043 & $0.3198-0.7953$ & 2.945 & 0.0032 \\
\hline PG1 & 0.5773 & $0.3619-0.9211$ & 2.305 & 0.0212 \\
\hline PG2 & 0.8926 & $0.5102-1.5616$ & 0.398 & 0.6906 \\
\hline PG3 & 2.3196 & $1.5051-3.5748$ & 3.813 & 0.0001 \\
\hline PG6 & 1.1339 & $0.0582-22.1005$ & 0.083 & 0.9339 \\
\hline PG7 & 2.9426 & $0.3889-22.2638$ & 1.045 & 0.2959 \\
\hline 1a & 0.8088 & $0.0386-16.9574$ & 0.137 & 0.8913 \\
\hline 1b & 0.6867 & $0.3942-1.1961$ & 1.328 & 0.1843 \\
\hline 2a & 1.9329 & $1.1712-3.1900$ & 2.578 & 0.0099 \\
\hline 2b & 2.2614 & $1.1117-4.5997$ & 2.252 & 0.0243 \\
\hline 3a & 0.8926 & $0.5102-1.5616$ & 0.398 & 0.6906 \\
\hline 4a & 0.7946 & $0.3230-1.9548$ & 0.501 & 0.6167 \\
\hline $5 \mathrm{~b}$ & 0.4798 & $0.0495-4.6540$ & 0.633 & 0.5264 \\
\hline 6 & 0.4829 & $0.3072-0.7590$ & 3.155 & 0.0016 \\
\hline $7 \mathrm{a}$ & 0.6029 & $0.2399-1.5151$ & 1.076 & 0.2818 \\
\hline Y & 1.46 & $0.0781-27.3032$ & 0.253 & 0.8001 \\
\hline X & 1.6157 & $0.2048-12.7458$ & 0.455 & 0.6489 \\
\hline
\end{tabular}


bioRxiv preprint doi: https://doi.org/10.1101/2021.06.09.447709; this version posted June 10, 2021. The copyright holder for this preprint (which was not certified by peer review) is the author/funder, who has granted bioRxiv a license to display the preprint in perpetuity. It is made available under aCC-BY 4.0 International license.

667 Table S4.

668

669 Details of serotype determining genes facilitating S. flexneri $(n=72)$ serotype switching.

670

671

672 See separate Excel file 
673 Table S5.

674 BEAST estimated timeframe for serotype switching among $S$. flexneri PG3 isolates.

675

\begin{tabular}{|c|c|c|c|c|c|}
\hline $\begin{array}{l}\text { Switch } \\
\text { ID }^{\#}\end{array}$ & Subclade $^{\text {II }}$ & $\begin{array}{l}\text { Serotype } \\
\text { change }\end{array}$ & $\begin{array}{l}\text { Molecular } \\
\text { serotype gene } \\
\text { detected }^{\&}\end{array}$ & $\begin{array}{l}\text { Median branch } \\
\text { length (days) }\end{array}$ & $\begin{array}{l}95 \% \text { HPD branch } \\
\text { length (days) }\end{array}$ \\
\hline 3 & $\mathrm{~A}$ & $2 \mathrm{a} \rightarrow \mathrm{Y}$ & - & 159 & $16-344$ \\
\hline 2 & $\mathrm{~A}$ & $2 a \rightarrow 7 a$ & gtrII & 154 & $27-307$ \\
\hline 1 & A & $2 a \rightarrow 2 b$ & gtrII, gtrX & 7203 & $4792-10009$ \\
\hline 7 & B & $2 b \rightarrow 5 b$ & gtrII, gtrX & 348 & $244-479$ \\
\hline 6 & B & $2 b \rightarrow 5 b$ & gtrII, gtrX & 254 & $134-491$ \\
\hline 5 & B & $2 \mathrm{~b} \rightarrow 1 \mathrm{~b}$ & $\begin{array}{l}\text { gtrI, gtrII, } \\
\text { Oaclb }\end{array}$ & 4888 & $2962-7114$ \\
\hline 4 & $\mathrm{~B}$ & $2 b \rightarrow X$ & gtrX, gtrll & 10206 & $5494-15408$ \\
\hline
\end{tabular}

677 Footnotes:

$678 \quad$ \# Serotype switching event labelled according to Fig S8

679 "Phylogenetic subclade isolate belong to

680 \& Presence of serotype determining genes, as detected by ShigaTyper, - indicates no genes were detected.

681 \$ Phylogenetic branch length represents divergence time, predicted by BEAST and inferred from a time682 scaled tree. 
Table S6.

An overview of the protein modelling. Table includes information about the antigen candidates modelled, the range of residues the proteins were modelled over, homologues used in template modelling and the QMEAN method and score.

\begin{tabular}{|l|l|l|l|l|l|l|l|l|l|}
\hline Species & $\begin{array}{l}\text { Antigen } \\
\text { candidates }\end{array}$ & Phylogroup & Serotype & Start & Finish & Homolog & $\begin{array}{l}\text { Sequence } \\
\text { Identity }\end{array}$ & $\begin{array}{l}\text { Qverage local } \\
\text { QMEAN score }\end{array}$ \\
\hline S. flexneri & OmpA & PG3 & $5 \mathrm{~A}$ & 22 & 216 & 1 QJP & $93 \%$ & QMEANDisCo & 0.46 \\
\hline S. flexneri & OmpA & PG3 & $5 \mathrm{~A}$ & 349 & 490 & $1 \mathrm{R} 1 \mathrm{M}$ & $41 \%$ & QMEANDisCo & 0.31 \\
\hline S. flexneri & SigA & PG3 & $2 \mathrm{~A}$ & 56 & 997 & $3 \mathrm{SZE}$ & $44 \%$ & QMEANDisCo & 0.71 \\
\hline S. flexneri & SigA & PG3 & $2 \mathrm{~A}$ & 998 & 1262 & $2 \mathrm{QOM}$ & $85 \%$ & QMEANDisCo & 0.83 \\
\hline S. flexneri & IcsP & PG3 & $5 \mathrm{~A}$ & 21 & 315 & $1 \mathrm{I} 78$ & $60 \%$ & QMEANDisCo & 0.81 \\
\hline S. flexneri & IpaB & PG3 & $5 \mathrm{~A}$ & 1 & 222 & $3 \mathrm{U} 0 \mathrm{C}$ & $76 \%$ & QMEANBrane & 0.83 \\
\hline S. flexneri & IpaB & PG3 & $5 \mathrm{~A}$ & 223 & 553 & $3 \mathrm{WXX}$ & $19 \%$ & QMEANBrane & 0.79 \\
\hline S. flexneri & IpaC & PG3 & $5 \mathrm{~A}$ & 1 & 363 & - & - & QMEANBrane & 0.85 \\
\hline S. flexneri & IpaD & PG3 & $5 \mathrm{~A}$ & 1 & 332 & $3 \mathrm{R} 9 \mathrm{~V}$ & $100 \%$ & QMEANBrane & 0.80 \\
\hline
\end{tabular}




\section{Table S7.}

2 Details of amino acid variants identified for the six antigen candidates among S. flexneri isolates from

3 GEMS. Table includes variant type, variant location, reference and alternative variant, and energy score of 4 the variant as predicted by premPS.

5

6 See separate Excel file 


\section{Reference}

1. Khalil IA, Troeger C, Blacker BF, Rao PC, Brown A, Atherly DE, et al. Morbidity and mortality due to shigella and enterotoxigenic Escherichia coli diarrhoea: the Global Burden of Disease Study 19902016. The Lancet infectious diseases. 2018;18(11):1229-40.

2. Kotloff KL, Nataro JP, Blackwelder WC, Nasrin D, Farag TH, Panchalingam S, et al. Burden and aetiology of diarrhoeal disease in infants and young children in developing countries (the Global Enteric Multicenter Study, GEMS): a prospective, case-control study. Lancet. 2013;382(9888):209-22.

3. Liu J, Platts-Mills JA, Juma J, Kabir F, Nkeze J, Okoi C, et al. Use of quantitative molecular diagnostic methods to identify causes of diarrhoea in children: a reanalysis of the GEMS case-control study. Lancet. 2016;388(10051):1291-301.

4. Kotloff KL, Riddle MS, Platts-Mills JA, Pavlinac P, Zaidi AKM. Shigellosis. Lancet. 2018;391(10122):801-12.

5. Shrivastava SR, Shrivastava PS, Ramasamy J. World health organization releases global priority list of antibiotic-resistant bacteria to guide research, discovery, and development of new antibiotics. Journal of Medical Society. 2018;32(1):76.

6. Barry EM, Pasetti MF, Sztein MB, Fasano A, Kotloff KL, Levine MM. Progress and pitfalls in Shigella vaccine research. Nat Rev Gastroenterol Hepatol. 2013;10(4):245-55.

7. Cohen D, Green MS, Block C, Slepon R, Ofek I. Prospective study of the association between serum antibodies to lipopolysaccharide $\mathrm{O}$ antigen and the attack rate of shigellosis. Journal of clinical microbiology. 1991;29(2):386-9.

8. Ferreccio C, Prado V, Ojeda A, Cayyazo M, Abrego P, Guers L, et al. Epidemiologic patterns of acute diarrhea and endemic Shigella infections in children in a poor periurban setting in Santiago, Chile. Am J Epidemiol. 1991;134(6):614-27.

9. Formal SB, Oaks EV, Olsen RE, Wingfield-Eggleston M, Snoy PJ, Cogan JP. Effect of prior infection with virulent Shigella flexneri 2a on the resistance of monkeys to subsequent infection with Shigella sonnei. J Infect Dis. 1991;164(3):533-7.

10. Kotloff KL, Nataro JP, Losonsky GA, Wasserman SS, Hale TL, Taylor DN, et al. A modified Shigella volunteer challenge model in which the inoculum is administered with bicarbonate buffer: clinical experience and implications for Shigella infectivity. Vaccine. 1995;13(16):1488-94.

11. Levine MM, Kotloff KL, Barry EM, Pasetti MF, Sztein MB. Clinical trials of Shigella vaccines: two steps forward and one step back on a long, hard road. Nat Rev Microbiol. 2007;5(7):540-53.

12. Ashkenazi S, Cohen D. An update on vaccines against Shigella. Ther Adv Vaccines. 2013;1(3):113-23.

13. Talaat KR, Alaimo C, Martin P, Bourgeois AL, Dreyer AM, Kaminski RW, et al. Human challenge study with a Shigella bioconjugate vaccine: Analyses of clinical efficacy and correlate of protection. EBioMedicine. 2021;66:103310.

14. Passwell JH, Ashkenazi S, Banet-Levi Y, Ramon-Saraf R, Farzam N, Lerner-Geva L, et al. Agerelated efficacy of Shigella O-specific polysaccharide conjugates in 1-4-year-old Israeli children. Vaccine. 2010;28(10):2231-5. 15. Turbyfill KR, Kaminski RW, Oaks EV. Immunogenicity and efficacy of highly purified invasin complex vaccine from Shigella flexneri 2a. Vaccine. 2008;26(10):1353-64. 
16. Martinez-Becerra FJ, Kissmann JM, Diaz-McNair J, Choudhari SP, Quick AM, Mellado-Sanchez $\mathrm{G}$, et al. Broadly protective Shigella vaccine based on type III secretion apparatus proteins. Infect Immun. 2012;80(3):1222-31.

17. Davies MR, McIntyre L, Mutreja A, Lacey JA, Lees JA, Towers RJ, et al. Atlas of group A streptococcal vaccine candidates compiled using large-scale comparative genomics. Nat Genet. 2019;51(6):1035-43.

18. Telford JL. Bacterial genome variability and its impact on vaccine design. Cell Host Microbe.

19. Livio S, Strockbine NA, Panchalingam S, Tennant SM, Barry EM, Marohn ME, et al. Shigella isolates from the global enteric multicenter study inform vaccine development. Clin Infect Dis. 2014;59(7):933-41.

20. Connor TR, Barker CR, Baker KS, Weill FX, Talukder KA, Smith AM, et al. Species-wide whole genome sequencing reveals historical global spread and recent local persistence in Shigella flexneri. Elife. 2015;4:e07335.

21. Holt KE, Baker S, Weill FX, Holmes EC, Kitchen A, Yu J, et al. Shigella sonnei genome sequencing and phylogenetic analysis indicate recent global dissemination from Europe. Nat Genet. 2012;44(9):1056-9.

22. Njamkepo E, Fawal N, Tran-Dien A, Hawkey J, Strockbine N, Jenkins C, et al. Global phylogeography and evolutionary history of Shigella dysenteriae type 1. Nat Microbiol. 2016;1:16027. 23. Kania DA, Hazen TH, Hossain A, Nataro JP, Rasko DA. Genome diversity of Shigella boydii. Pathog Dis. 2016;74(4):ftw027. population structure and genotyping framework for genomic surveillance of the major dysentery pathogen, Shigella sonnei. Nature Communications. 2021;12(1):2684.

25. Sahl JW, Morris CR, Emberger J, Fraser CM, Ochieng JB, Juma J, et al. Defining the phylogenomics of Shigella species: a pathway to diagnostics. J Clin Microbiol. 2015;53(3):951-60. 26. von Seidlein L, Kim DR, Ali M, Lee H, Wang X, Thiem VD, et al. A multicentre study of Shigella diarrhoea in six Asian countries: disease burden, clinical manifestations, and microbiology. PLoS Med. 2006;3(9):e353.

78 27. Ye C, Lan R, Xia S, Zhang J, Sun Q, Zhang S, et al. Emergence of a new multidrug-resistant serotype X variant in an epidemic clone of Shigella flexneri. J Clin Microbiol. 2010;48(2):419-26. Shigella flexneri. Trends Microbiol. 2000;8(1):17-23.

29. Sun Q, Knirel YA, Lan R, Wang J, Senchenkova SN, Jin D, et al. A novel plasmid-encoded serotype conversion mechanism through addition of phosphoethanolamine to the O-antigen of Shigella flexneri. PLoS One. 2012;7(9):e46095.

30. Weinberger DM, Malley R, Lipsitch M. Serotype replacement in disease after pneumococcal vaccination. Lancet. 2011;378(9807):1962-73.

31. Brueggemann AB, Pai R, Crook DW, Beall B. Vaccine escape recombinants emerge after pneumococcal vaccination in the United States. PLoS Pathog. 2007;3(11):e168.

32. McVicker G, Tang CM. Deletion of toxin-antitoxin systems in the evolution of Shigella sonnei as a host-adapted pathogen. Nat Microbiol. 2016;2:16204.

33. Garcia-Beltran WF, Lam EC, St Denis K, Nitido AD, Garcia ZH, Hauser BM, et al. Multiple SARS-CoV-2 variants escape neutralization by vaccine-induced humoral immunity. Cell. 2021.

34. Zhou D, Dejnirattisai W, Supasa P, Liu C, Mentzer AJ, Ginn HM, et al. Evidence of escape of SARS-CoV-2 variant B.1.351 from natural and vaccine-induced sera. Cell. 2021. 
35. Mills JA, Buysse JM, Oaks EV. Shigella flexneri invasion plasmid antigens B and C: epitope location and characterization with monoclonal antibodies. Infect Immun. 1988;56(11):2933-41. 36. Turbyfill KR, Mertz JA, Mallett CP, Oaks EV. Identification of epitope and surface-exposed domains of Shigella flexneri invasion plasmid antigen D (IpaD). Infect Immun. 1998;66(5):1999-2006. 37. Czerkinsky C, Kim DW. Shigella protein antigens and methods. US Patent 8168203; 2012. 38. Pore D, Mahata N, Pal A, Chakrabarti MK. Outer membrane protein A (OmpA) of Shigella flexneri 2a, induces protective immune response in a mouse model. PLoS One. 2011;6(7):e22663. 39. Organization WH. Guidelines for the control of shigellosis, including epidemics due to Shigella dysenteriae type 1. 2005.

40. Chung The H, Baker S. Out of Asia: the independent rise and global spread of fluoroquinoloneresistant Shigella. Microb Genom. 2018;4(4).

41. Sadouki Z, Day MR, Doumith M, Chattaway MA, Dallman TJ, Hopkins KL, et al. Comparison of phenotypic and WGS-derived antimicrobial resistance profiles of Shigella sonnei isolated from cases of diarrhoeal disease in England and Wales, 2015. J Antimicrob Chemother. 2017;72(9):2496-502. 42. Baker KS, Dallman TJ, Ashton PM, Day M, Hughes G, Crook PD, et al. Intercontinental dissemination of azithromycin-resistant shigellosis through sexual transmission: a cross-sectional study. Lancet Infect Dis. 2015;15(8):913-21.

43. Williams PCM, Berkley JA. Guidelines for the treatment of dysentery (shigellosis): a systematic review of the evidence. Paediatr Int Child Health. 2018;38(sup1):S50-S65.

44. Chung The H, Rabaa MA, Pham Thanh D, De Lappe N, Cormican M, Valcanis M, et al. South Asia as a Reservoir for the Global Spread of Ciprofloxacin-Resistant Shigella sonnei: A Cross-Sectional Study. PLoS Med. 2016;13(8):e1002055.

45. Chung The H, Boinett C, Pham Thanh D, Jenkins C, Weill FX, Howden BP, et al. Dissecting the molecular evolution of fluoroquinolone-resistant Shigella sonnei. Nat Commun. 2019;10(1):4828.

46. Ingle DJ, Levine MM, Kotloff KL, Holt KE, Robins-Browne RM. Dynamics of antimicrobial resistance in intestinal Escherichia coli from children in community settings in South Asia and subSaharan Africa. Nat Microbiol. 2018;3(9):1063-73.

47. Makoni M. Africa's \$100-million Pathogen Genomics Initiative. The Lancet Microbe. 2020;1(8):e318.

48. AMR NGHRUoGSo. Whole-genome sequencing as part of national and international surveillance programmes for antimicrobial resistance: a roadmap. BMJ Global Health. 2020;5(11):e002244.

49. Perez-Sepulveda BM, Heavens D, Pulford CV, Predeus AV, Low R, Webster H, et al. An accessible, efficient and global approach for the large-scale sequencing of bacterial genomes. BioRxiv. 2020.

50. Bolger AM, Lohse M, Usadel B. Trimmomatic: a flexible trimmer for Illumina sequence data. Bioinformatics. 2014;30(15):2114-20.

51. Ewels P, Magnusson M, Lundin S, Kaller M. MultiQC: summarize analysis results for multiple tools and samples in a single report. Bioinformatics. 2016;32(19):3047-8.

134 52. Li H. Aligning sequence reads, clone sequences and assembly contigs with BWA-MEM. arXiv 135 preprint arXiv:13033997. 2013.

136 53. Li H, Handsaker B, Wysoker A, Fennell T, Ruan J, Homer N, et al. The Sequence

137 Alignment/Map format and SAMtools. Bioinformatics. 2009;25(16):2078-9.

138 54. Garcia-Alcalde F, Okonechnikov K, Carbonell J, Cruz LM, Gotz S, Tarazona S, et al. Qualimap: 139 evaluating next-generation sequencing alignment data. Bioinformatics. 2012;28(20):2678-9. 
55. Arndt D, Grant JR, Marcu A, Sajed T, Pon A, Liang Y, et al. PHASTER: a better, faster version of the PHAST phage search tool. Nucleic Acids Res. 2016;44(W1):W16-21.

56. Quinlan AR. BEDTools: The Swiss-Army Tool for Genome Feature Analysis. Curr Protoc Bioinformatics. 2014;47:11 2 1-34.

57. Croucher NJ, Page AJ, Connor TR, Delaney AJ, Keane JA, Bentley SD, et al. Rapid phylogenetic analysis of large samples of recombinant bacterial whole genome sequences using Gubbins. Nucleic Acids Res. 2015;43(3):e15.

58. Nguyen LT, Schmidt HA, von Haeseler A, Minh BQ. IQ-TREE: a fast and effective stochastic algorithm for estimating maximum-likelihood phylogenies. Mol Biol Evol. 2015;32(1):268-74.

59. Letunic I, Bork P. Interactive Tree Of Life (iTOL) v4: recent updates and new developments. Nucleic Acids Res. 2019;47(W1):W256-W9.

60. Rambaut A, Lam TT, Max Carvalho L, Pybus OG. Exploring the temporal structure of heterochronous sequences using TempEst (formerly Path-O-Gen). Virus Evol. 2016;2(1):vew007. 61. Bouckaert R, Heled J, Kuhnert D, Vaughan T, Wu CH, Xie D, et al. BEAST 2: a software platform for Bayesian evolutionary analysis. PLoS Comput Biol. 2014;10(4):e1003537.

62. Bouckaert RR, Drummond AJ. bModelTest: Bayesian phylogenetic site model averaging and model comparison. BMC Evol Biol. 2017;17(1):42.

63. Rambaut A, Drummond AJ, Xie D, Baele G, Suchard MA. Posterior Summarization in Bayesian Phylogenetics Using Tracer 1.7. Syst Biol. 2018;67(5):901-4.

64. Bouckaert R, Vaughan TG, Barido-Sottani J, Duchene S, Fourment M, Gavryushkina A, et al. BEAST 2.5: An advanced software platform for Bayesian evolutionary analysis. PLoS Comput Biol. 2019;15(4):e1006650.

65. Wick RR, Judd LM, Gorrie CL, Holt KE. Unicycler: resolving bacterial genome assemblies from short and long sequencing reads. PLoS computational biology. 2017;13(6):e1005595.

66. Gurevich A, Saveliev V, Vyahhi N, Tesler G. QUAST: quality assessment tool for genome assemblies. Bioinformatics. 2013;29(8):1072-5.

67. Seemann T. Prokka: rapid prokaryotic genome annotation. Bioinformatics. 2014;30(14):2068-9.

68. Page AJ, Cummins CA, Hunt M, Wong VK, Reuter S, Holden MT, et al. Roary: rapid large-scale prokaryote pan genome analysis. Bioinformatics. 2015;31(22):3691-3.

69. Wu Y, Lau HK, Lee T, Lau DK, Payne J. In Silico Serotyping Based on Whole-Genome Sequencing Improves the Accuracy of Shigella Identification. Appl Environ Microbiol. 2019;85(7).

70. Inouye M, Dashnow H, Raven LA, Schultz MB, Pope BJ, Tomita T, et al. SRST2: Rapid genomic surveillance for public health and hospital microbiology labs. Genome Med. 2014;6(11):90.

71. Altschul SF, Madden TL, Schaffer AA, Zhang J, Zhang Z, Miller W, et al. Gapped BLAST and PSI-BLAST: a new generation of protein database search programs. Nucleic Acids Res. 1997;25(17):3389-402.

72. Larsson A. AliView: a fast and lightweight alignment viewer and editor for large datasets. Bioinformatics. 2014;30(22):3276-8.

73. Hildebrand A, Remmert M, Biegert A, Soding J. Fast and accurate automatic structure prediction with HHpred. Proteins. 2009;77 Suppl 9:128-32.

74. Zimmermann L, Stephens A, Nam SZ, Rau D, Kubler J, Lozajic M, et al. A Completely Reimplemented MPI Bioinformatics Toolkit with a New HHpred Server at its Core. J Mol Biol. 2018;430(15):2237-43.

75. Song Y, DiMaio F, Wang RY, Kim D, Miles C, Brunette T, et al. High-resolution comparative modeling with RosettaCM. Structure. 2013;21(10):1735-42. 
185 76. Yang J, Anishchenko I, Park H, Peng Z, Ovchinnikov S, Baker D. Improved protein structure

186 prediction using predicted interresidue orientations. Proceedings of the National Academy of Sciences.

187 2020;117(3):1496-503.

188 77. Studer G, Rempfer C, Waterhouse AM, Gumienny R, Haas J, Schwede T. QMEANDisCo-

189 distance constraints applied on model quality estimation. Bioinformatics. 2020;36(8):2647.

190 78. Studer G, Biasini M, Schwede T. Assessing the local structural quality of transmembrane protein

191 models using statistical potentials (QMEANBrane). Bioinformatics. 2014;30(17):i505-11.

192 79. Chen Y, Lu H, Zhang N, Zhu Z, Wang S, Li M. PremPS: Predicting the impact of missense

193 mutations on protein stability. PLoS Comput Biol. 2020;16(12):e1008543.

194 80. Feldgarden M, Brover V, Haft DH, Prasad AB, Slotta DJ, Tolstoy I, et al. Validating the

195 AMRFinder Tool and Resistance Gene Database by Using Antimicrobial Resistance Genotype-Phenotype

196 Correlations in a Collection of Isolates. Antimicrob Agents Chemother. 2019;63(11).

197 81. Conway JR, Lex A, Gehlenborg N. UpSetR: an R package for the visualization of intersecting

198 sets and their properties. Bioinformatics. 2017;33(18):2938-40.

199 82. Hudzicki J. Kirby-Bauer disk diffusion susceptibility test protocol. 2009. 